\title{
A non-transitive relevant implication corresponding to classical logic consequence
}

\author{
Peter Verdée, Inge De Bal, Aleksandra Samonek
}

\begin{abstract}
In this paper we first develop a logic independent account of relevant implication. We propose a stipulative definition of what it means for a multiset of premises to relevantly L-imply a multiset of conclusions, where $\mathbf{L}$ is a Tarskian consequence relation: the premises relevantly imply the conclusions iff there is an abstraction of the pair 〈premises, conclusions〉 such that the abstracted premises $\mathbf{L}$-imply the abstracted conclusions and none of the abstracted premises or the abstracted conclusions can be omitted while still maintaining valid L-consequence.

Subsequently we apply this definition to the classical logic (CL) consequence relation to obtain NTR-consequence, i.e. the relevant CL-consequence relation in our sense, and develop a sequent calculus that is sound and complete w.r.t. relevant CL-consequence. We present a sound and complete sequent calculus for NTR. In a next step we add rules for an object language relevant implication to the sequent calculus. The object language implication reflects exactly the NTR-consequence relation. One can see the resulting logic $\mathbf{N T R} \rightarrow$ as a relevant logic in the traditional sense of the word.

By means of a translation to the relevant $\operatorname{logic} \mathbf{R}$, we show that the presented logic NTR is very close to relevance logics in the AndersonBelnap-Dunn-Routley-Meyer tradition. However, unlike usual relevant logics, NTR is decidable for the full language, Disjunctive Syllogism ( $A$ and $\neg A \vee B$ relevantly imply $B$ ) and Adjunction ( $A$ and $B$ relevantly imply $A \wedge B$ ) are valid, and neither Modus Ponens nor the Cut rule are admissible.
\end{abstract}

Australasian Journal of Logic (16:2) 2019, Article no. 1 


\section{Introduction}

In the recent history of logic there are few episodes as mathematically and philosophically rich as the development of relevance logics to avoid the counterintuitive properties (sometimes called 'paradoxes' or even 'fallacies' by relevance logicians) of material implication. Starting with Wilhelm Ackermann [1] and Alonzo Church [6] in the 1950s, many logicians have studied logical systems that aim to get rid of irrelevance in logic. Nuel Belnap and Alan Anderson (among many others) [2,3] have developed and thoroughly analyzed the best known relevance logics $\mathbf{E}$ and $\mathbf{R}$, for which Richard Routley/Sylvan and Bob Meyer $[16,15]$ have proposed a very interesting and intriguing possible world semantics with a ternary accessibility relation. For the technical work in this paper the encyclopedic article [8] suffices. Despite the beauty and importance of this thread of research, we think it is worthwhile to attempt a completely different road to relevance from a more pluralistic and conservative point of view. We want to look at a notion of relevance that is as close as possible to existing logics that have no relevance properties. We study the notion of relevant implication for existing consequence relations, and in particular for classical logic (CL). We aim to investigate the position that, whatever notion of logical consequence one endorses, one can coherently speak of and formalize the notion of relevant implication, without criticizing the underlying (non-relevant) consequence relation. We thus look for a notion of relevance that is complimentary and tailored to existing nonrelevant logics, and in this paper specifically to classical logic. $\mathbf{R}$ and $\mathbf{E}$ are not tailored for classical logic as illustrated by the rejection of Disjunctive Syllogism, which is very often (relevantly) used in classical reasoning contexts (as argued by [4]). At the end of this paper however, it will be shown that the here proposed relevant implication tailored for classical logic is actually not extremely different from the standard relevance logic $\mathbf{R}$ and could thus be seen as a contribution to the logical relevance research thread starting with Ackermann.

Let $\mathcal{W}$ be the set of formulas of a formal language $\mathcal{L}$. A (multiple conclusion) Tarskian consequence relation $\vdash$ (cf. [11]) is a relation in $\wp(\mathcal{W}) \times \wp(\mathcal{W})$ that is monotonic (i.e. if $\Gamma \vdash \Delta$, then $\Gamma \cup\{A\} \vdash \Delta$ and $\Gamma \vdash \Delta \cup\{A\}$ ), transitive (i.e. if $\Gamma \vdash \Delta \cup\{A\}$ and $\Gamma^{\prime} \cup\{A\} \vdash \Delta^{\prime}$, then $\Gamma \cup \Gamma^{\prime} \vdash \Delta \cup \Delta^{\prime}$ ), reflexive (i.e. $\{A\} \vdash\{A\}$ ), and formal (i.e. closed under Uniform Substitution). Remark that all axiomatizable consequence relations are Tarskian. Semantically, Tarskian consequence relations can usually be characterized as follows:

Australasian Journal of Logic (16:2) 2019 Article no. 1 
$\Delta$ is a consequence of $\Gamma$ iff at least one of the members of $\Delta$ is verified by each model that verifies all members of $\Gamma$, where the precise meaning of the expressions 'model' and 'is verified by' differs from logic to logic. Consider any $\operatorname{logic} \mathbf{L}$ that defines a Tarskian consequence relation $\vdash_{\mathbf{L}}$.

As an example, consider the $\mathbf{C L}$-consequence relation with multiple conclusions. Remember that in multiple conclusion consequence relations at least one of the conclusions should be true in all models in which all premises are true. So e.g.

$$
\begin{gathered}
p, \neg p \vee q \vdash_{\mathbf{C L}} q, r \\
\vdash_{\mathbf{C L}} p, \neg p \\
p, q \vdash_{\mathbf{C L}} q, r \\
p, \neg p \vdash_{\mathbf{C L}} \\
s, p \vee q \vdash_{\mathbf{C L}} p, q, r
\end{gathered}
$$

but

$$
\begin{gathered}
p, \neg p \vee q \nvdash_{\mathbf{C L}} r, s \\
\nvdash_{\mathbf{C L}} p, \neg q \\
p, q \nvdash_{\mathbf{C L}} r \\
p, \neg q \nvdash_{\mathbf{C L}} \\
s, p \vee q \nvdash_{\mathbf{C L}} p, r
\end{gathered}
$$

In what follows regular Greek capital letters-possibly with superscripts, subscripts and accents-(e.g. $\left.\Gamma, \Delta^{\prime}, \Theta_{3}\right)$ will denote subsets of $\mathcal{W}$. Bold Greek capitals-possibly with superscripts, subscripts and accents-(e.g. Г, $\boldsymbol{\Delta}^{\prime}, \boldsymbol{\Theta}_{\mathbf{3}}$ ) will denote multisets ${ }^{1}$ of formulas in $\mathcal{W}$. Where $\ddagger$ is a symbol, a set $\ddagger$-sequent is a statement $\Gamma \ddagger \Delta$ and a multiset $\ddagger$-sequent is a statement $\boldsymbol{\Gamma} \ddagger \boldsymbol{\Delta}$. Multiset $\vdash_{\mathbf{L}}$-sequents will be said to be valid iff the set version of the same sequent (when only one copy is left of each element on both sides of the turnstyle) is valid.

For example, $p, q \vdash_{\mathbf{C L}} q \wedge r, s$ and $p, \neg q \vdash_{\mathbf{C L}}$ are (invalid) $\vdash_{\mathbf{C L}_{\mathbf{L}} \text {-sequents }}$ $p, p, p, r \vdash_{\mathbf{C L}}$ and $r, r, r \vdash_{\mathbf{C L}} p, p, \neg p$ are multiset $\vdash_{\mathbf{C L}}$-sequents (the first invalid, the second valid). If there is no confusion about the central symbol of

\footnotetext{
${ }^{1} \mathrm{~A}$ multiset is like a set, but distinguishes for each element the number of copies of that element. The union of two multisets has the same elements as the union of the elements of the two multisets. The number of copies of each element of the union multiset is the sum of the number of its copies in the first multiset and the number of its copies in the second.
}

Australasian Journal of Logic (16:2) 2019 Article no. 1 
the sequent or about its being a mutiset sequent or not, we simply use the word 'sequent'.

Let's introduce our stipulative definition of $\mathbf{L}$-relevance and relevant $\mathbf{L}$ implication.

Definition 1. A valid multiset $\vdash_{\mathbf{L}}$-sequent is $\mathbf{L}$-relevant iff there is an $\mathbf{L}$ valid abstraction of this multiset sequent in which none of the premises or conclusions are redundant. We say that $\boldsymbol{\Gamma}$ relevantly $\mathbf{L}$-implies $\boldsymbol{\Delta}$ iff the sequent $\boldsymbol{\Gamma} \vdash_{\mathbf{L}} \boldsymbol{\Delta}$ is $\mathbf{L}$-relevantly valid.

In order to make this definition precise we need to give a definition of abstraction and redundancy in a valid $\mathbf{L}$-implication. We say that a multiset sequent $\boldsymbol{\Gamma}^{\prime} \vdash \boldsymbol{\Delta}^{\prime}$ is an abstraction of a multiset sequent $\boldsymbol{\Gamma} \vdash \boldsymbol{\Delta}$ iff the latter, or a version of the latter with more copies of some of its formulas, can be obtained from the former by one or more applications of Uniform Substitution, where Uniform Substitution is the rule that enables the replacement of every occurrence of a sentential letter (in the entire sequent) by a (primitive or complex) formula. Or, more formally:

Definition 2. The notion abstraction is recursively defined as follows.

- $\boldsymbol{\Gamma} \vdash \boldsymbol{\Delta}$ is an abstraction of $\boldsymbol{\Gamma} \vdash \boldsymbol{\Delta}$,

- $\boldsymbol{\Gamma} \cup\{A, A\} \vdash \boldsymbol{\Delta}$ is an abstraction of $\boldsymbol{\Gamma} \cup\{A\} \vdash \boldsymbol{\Delta}$,

- $\boldsymbol{\Gamma} \vdash \boldsymbol{\Delta} \cup\{A, A\}$ is an abstraction of $\boldsymbol{\Gamma} \vdash \boldsymbol{\Delta} \cup\{A\}$,

- $\boldsymbol{\Gamma}^{\prime} \vdash \boldsymbol{\Delta}^{\prime}$ is an abstraction of a multiset sequent $\boldsymbol{\Gamma} \vdash \boldsymbol{\Delta}$, where $\langle\Gamma, \Delta\rangle$ is the result of substituting every occurrence of a proposition letter in $\left\langle\Gamma^{\prime}, \Delta^{\prime}\right\rangle$ by one single formula,

- If $\boldsymbol{\Gamma}^{\prime \prime} \vdash \boldsymbol{\Delta}^{\prime \prime}$ is an abstraction of $\boldsymbol{\Gamma}^{\prime} \vdash \boldsymbol{\Delta}^{\prime}$ and $\boldsymbol{\Gamma}^{\prime} \vdash \boldsymbol{\Delta}^{\prime}$ is an abstraction of $\boldsymbol{\Gamma} \vdash \boldsymbol{\Delta}$, then $\boldsymbol{\Gamma}^{\prime \prime} \vdash \boldsymbol{\Delta}^{\prime \prime}$ is an abstraction of $\boldsymbol{\Gamma} \vdash \boldsymbol{\Delta}$, and

- nothing else is an abstraction.

Note that if an abstraction of a sequent is $\mathbf{L}$-valid, then the abstracted sequent is also $\mathbf{L}$-valid (by the formality of $\mathbf{L}$ ).

Definition 3. We say that a premise resp. a conclusion is redundant in an $\mathbf{L}$-valid sequent iff the sequent is still $\mathbf{L}$-valid after that premise resp. that conclusion is removed from the premises resp. conclusions of the sequent.

Australasian Journal of Logic (16:2) 2019 Article no. 1 


\begin{tabular}{l|l} 
sequent & is an abstraction of sequent \\
\hline$p, \neg p \vdash_{\mathbf{C L}}$ & $p \wedge r, \neg(p \wedge r) \vdash_{\mathbf{C L}}$ \\
$\vdash_{\mathbf{C L}} q, \neg q$ & $\vdash_{\mathbf{C L}} p \wedge \neg p, \neg(p \wedge \neg p)$ \\
$p, \neg p \vee q \vdash_{\mathbf{C L}} q$ & $p, \neg p \vee p \vdash_{\mathbf{C L}} p$ \\
$p \vee q \vdash_{\mathbf{C L}} p, q$ & $(p \wedge r) \vee(p \wedge r) \vdash_{\mathbf{C L}} p \wedge r, p \wedge r$ \\
$p, q \vdash_{\mathbf{C L}} p \wedge q$ & $p, \neg p \vdash_{\mathbf{C L}} p \wedge \neg p$ \\
$\neg(p \vee q) \vdash_{\mathbf{C L}} \neg p \wedge \neg q$ & $\neg(p \vee p) \vdash_{\mathbf{C L}} \neg p \wedge \neg p$ \\
$p, q \vdash p \wedge_{\mathbf{C L}}(q \wedge r), \neg r$ & $p \wedge q, p \wedge q \vdash_{\mathbf{C L}}(p \wedge q) \wedge((p \wedge q) \wedge(p \wedge q)), \neg(p \wedge q)$ \\
$p, q \vdash p \wedge q$ & $p, p \vdash p \wedge p$ \\
$p \vee q \vdash p, q$ & $p \vee p \vdash p, p$ \\
$p \vee p \vdash p, p$ & $p \vee p \vdash p$
\end{tabular}

Table 1: examples of abstractions.

For example, the following are valid $\vdash_{\mathbf{C L}}$-sequents in which no premises or conclusions are redundant (the reader can verify that the sequents become invalid as soon as one removes one formula in the sequent):

$$
\begin{gathered}
p, \neg p \vdash_{\mathbf{C L}} \\
\vdash_{\mathbf{C L}} q, \neg q \\
p, \neg p \vee q \vdash_{\mathbf{C L}} q \\
p \vee q \vdash_{\mathbf{C L}} p, q \\
p, q \vdash_{\mathbf{C L}} p \wedge q \\
\neg(p \vee q) \vdash_{\mathbf{C L}} \neg p \wedge \neg q \\
p, q \vdash_{\mathbf{C L}} p \wedge(q \wedge r), \neg r
\end{gathered}
$$

Whatever formula one adds to these sequents, it will be redundant for the validity of the consequent. For example,

$$
\begin{gathered}
q \text { is redundant in } p, \neg p \vdash_{\mathbf{C L}} q \\
r \text { is redundant in } r \vdash_{\mathbf{C L}} q, \neg q \\
\neg p \text { is redundant in } p, \neg p \vee q \vdash_{\mathbf{C L}} q, \neg p \\
\text { premise } q \text { is redundant in } q, p \vee \vdash_{\mathbf{C L}} p, q .
\end{gathered}
$$

To illustrate what an abstraction of a sequent is, see Table 1.

Observe that the abstractions are multisets: premises and conclusions may occur more than once. In each of the above examples the multiset sequents are CL-relevant, because there is a CL-valid abstraction without redundancy. This does not imply absence of redundancy. Consider that, in Australasian Journal of Logic (16:2) 2019 Article no. 1 
every valid multiset sequent in which some premises/conclusions occur more than once, at least one copy of these premises/conclusions is redundant. The following examples illustrate this redundancy.

In the relevant $\vdash_{\mathbf{C L}} p \wedge \neg p, \neg(p \wedge \neg p)$, the conclusion $p \wedge \neg p$ is redundant because also $\vdash_{\mathbf{C L}} \neg(p \wedge \neg p)$ is valid, but the abstraction $\vdash_{\mathbf{C L}} q, \neg q$ does not contain redundancy.

In the relevant $p, \neg p \vee p \vdash_{\mathbf{C L}} p$, the premise $\neg p \vee p$ is redundant because it is a tautology, but the abstraction $p, \neg p \vee q \vdash_{\mathbf{C L}} q$ does not contain redundancy.

In the relevant $(p \wedge r) \vee(p \wedge r) \vdash_{\mathbf{C L}} p \wedge r, p \wedge r$ one of the copies of the conclusion $p \wedge r$ is redundant, but the abstraction $q \vee(p \wedge r) \vdash_{\mathbf{C L}} q, p \wedge r$ does not contain redundancy.

In the relevant $p, \neg p \vdash p \wedge \neg p$ the conclusion is redundant because it is a contradiction, but the abstraction $p, \neg q \vdash p \wedge \neg q$ does not contain redundancy.

In the relevant $p \wedge q, p \wedge q \vdash_{\mathbf{C L}}(p \wedge q) \wedge((p \wedge q) \wedge(p \wedge q)), \neg(p \wedge q)$, one of the copies of the premise and the second conclusion are both redundant, but the abstraction $s, t \vdash_{\mathbf{C L}} r \wedge(s \wedge t), \neg r$ does not contain redundancy.

The idea behind this stipulative definition is that, intuitively, we can say that premises relevantly imply conclusions iff the combination of all the premises gives us enough grounds to formally argue for one of the conclusions, in such a way that each involved statement is useful to establish the validity relation (i.e. none of the formulas are redundant or, put differently, can be removed or replaced by an arbitrary formula, without jeopardizing validity). In other words: there is a formal argument for the conclusion using each of the premises. We speak of 'formally argue' and a 'formal argument' because it is the form of the argument that needs to be non-redundant. The concrete instance may contain redundancies. The notions 'argument' and 'used' or 'useful' here and in what follows seems to be proof theoretic notions, but it is not meant that way: a formal argument is here meant as nothing more than a generally valid (abstract) syllogism, principle of reasoning, or admissible inference rule, in any possible proof system one would develop for the consequence relation. When we say 'useful' we merely mean that it is not redundant in the employed principle of reasoning. We admit that a proof theoretic account of these notions would make more sense, but here we want to stay as general as possible, beyond any concrete logic or proof theory.

Australasian Journal of Logic (16:2) 2019 Article no. 1 
For example, $p, \neg p \vee p \vdash_{\mathbf{C L}} p$ is relevant because there exists a formal argument of which it is a token (viz. $A, \neg A \vee B \vdash_{\mathbf{C L}} B$ ) that does not contain redundancies. So, in some sense, all premises and conclusion of $p, \neg p \vee p \vdash_{\mathbf{C L}} p$ are useful because they are indispensable in (at least) one way to formally argue for the sequent. One more example:

$\vdash_{\mathbf{C L}} p \vee \neg p, p \vee \neg p$ is relevant because $\vdash_{\mathbf{C L}} A \vee \neg B, B \vee C$ is non-redundant, not because $\vdash_{\mathbf{C L}} A \vee \neg A$ is.

Although this is a stipulative definition of relevant validity, we conjecture that this is one of the ways in which the expressions 'together entail', 'follows from', 'together imply' etc. are used in natural language (e.g. in mathematics or science papers). It seems fair to assume that whenever a scientist claims that statement (1), (2) and (3) together entail/imply (4) or that (4) follows from (1), (2), and (3), she means that (4) is relevantly implied by (1), (2) and (3) in the above sense, i.e. that she has a formal argument for (4) from (1)-(3), in which all of (1)-(4) are effectively useful.

The advantages of this definition are the following. First, in this sense of relevance, relevant validity is closed under Uniform Substitution and is thus a formal relation. For example, because $p, \neg p \vee q \vdash_{\mathbf{C L}} q$ is relevant, every instance of $A, \neg A \vee B \vdash_{\mathbf{C L}} B$ is also relevant. Secondly, it can be applied to every Tarskian consequence relation and so does not presuppose any specific view on logic or on the meaning of the involved connectives. Thirdly, it is implicitly based on a reasonable notion of usefulness in an argument. If premises relevantly imply a conclusion, then there is a valid argument for the conclusion which really uses each of the premises. If the conclusion is moreover non-tautological, then the converse conditional also holds. Fourth, given that there is a sense in which none of the involved formulas of a relevant valid sequent are redundant, there must be a real connection between the premises and the conclusions. It is due to the premises and the other conclusions (or, in absence of premises, only the other conclusions) that we can obtain any particular conclusion (that particular conclusion is not obtained independently).

A possible criticism may be that, given this definition, even though premises relevantly imply conclusions, some of the premises and conclusions may be redundant (see examples above). Remember that it suffices that an abstraction of the (multiset) sequent is non-redundant, not that it is nonredundant itself. This, however, is unavoidable if one wants to develop a formal account of relevance. One can always instantiate premises of a relevant

Australasian Journal of Logic (16:2) 2019 Article no. 1 
form of an argument in such a way that a conclusion becomes a contradiction or that a premise becomes a tautology (and so automatically redundant). In other words, if one has a formal proof, based on formal rules, that some conclusion follows from some premises in a relevant way, one can always redo the proof, by uniform substitution, in such a way that the conclusion becomes a contradiction (unless the conclusion was a tautology before substitution, but then the premises were already redundant). Such a conclusion is by definition redundant (if a contradiction follows, anything follows).

One could in principle avoid this redundancy by using a non-formal notion of relevant validity along the following lines: premises informally relevantly entail conclusions if none of the premises or conclusions are redundant for validity. But this does not solve much. Given classical logic one would then say that $p \wedge q$ informally relevantly implies $p$. But as soon as we instantiate this sequent redundancies may show up again. For example, take $p$ to be 'object $a$ is round' and $q$ to be 'object $a$ is square'. This object being a round square should then informally relevantly imply it being round. But informally anything follows from the object being round and square (it is an informal/material inconsistency). So it being round is in some sense still redundant to the validity. If one also wants to eliminate cases like that, no (logically) relevant validities remain that are relevant independently of the context in which they are used. We conclude from this observation that, if context independent relevance makes any sense at all (and we think it does), it should be robust under applications of Uniform Substitution. A consequence of this is that non-redundancy of all premises and conclusions is too strong a criterion for relevant validity. Incidentally, consider that all standard accounts of relevance logic are also formal and so cannot avoid the same sort of redundancies. As an example, one can verify that $(p \rightarrow$ $(q \wedge p)) \rightarrow(p \rightarrow p)$ is a theorem of the relevance logic $\mathbf{R}$ although the consequent of this implication is itself already a theorem of $\mathbf{R}$.

Let us now investigate this general, logic independent definition for the concrete case of the logic $\mathbf{C L}$. It is clear that not every valid $\vdash_{\mathbf{C L}}$-sequent is relevant. As an example take: $p \vdash_{\mathbf{C L}} p, q$, where $q$ is redundant in each formal argument that grounds the validity of the sequent. Relevant validity is a much stronger property than logical consequence in general ${ }^{2}$.

\footnotetext{
${ }^{2}$ We use the concept 'logical consequence relation' here as it usually used in the literature, viz. as the relation of truth preservation in the Tarskian sense. We do not want to claim that the pre-theoretic notion of 'consequence' is anything like this. In fact it may well be that the latter notion is closer to what we call relevant implication than to truth
}

Australasian Journal of Logic (16:2) 2019 Article no. 1 
Let us see how the definition of relevant CL-implication works in practice. Do $p, \neg p \vee q$, and $(\neg q \vee p) \wedge p$ relevantly CL-imply $p$ ? Prima facie, one may think that it is not the case, because the second and third premise seem useless. But consider that $\langle p, \neg p \vee q,(\neg q \vee r) \wedge s, r\rangle$ is an abstraction of $\langle p, \neg p \vee q,(\neg q \vee p) \wedge p, p\rangle$, and moreover it holds non-redundantly that $p, \neg p \vee q,(\neg q \vee r) \wedge s \vdash_{\mathbf{C L}} r$. So $p$ is relevantly CL-implied by $p, \neg p \vee q$, and $(\neg q \vee p) \wedge p$ after all. Is this not evidence that the definition flags absurd consequences as relevant? We think this is not the case, for consider the following proof:

$1 p \quad$ PREM

$2 \neg p \vee q \quad$ PREM

$3 q \quad$ Disjunctive Syllogism; 1,2

$4 \quad(\neg q \vee p) \wedge p \quad$ PREM

$5 \neg q \vee p \quad$ Elimination of Conjunction; 4

$6 p \quad$ Disjunctive Syllogism; 3,5

In this proof all the premises are effectively used to obtain the conclusion. One could of course object that this is far from the most efficient proof. But do we want to stipulate that we can only claim that a conclusion is relevantly implied by premises if all the premises are used in the most efficient proof? That seems too restrictive. As soon as we really use the premises in the derivation of the conclusion, they are relevant for the conclusion.

Although the notion of relevance is based on Tarskian consequence relations, the set of relevant sequents does not constitute a Tarskian consequence relation. Obviously it is not monotonic: adding premises or conclusion easily makes a sequent irrelevant. But it is not transitive either. Consider premises $p$ and $q$ and conclusion $p$. Although $p$ and $q$ together relevantly CL-imply $p \wedge q$ and moreover $p \wedge q$ relevantly CL-implies $p$, it does not make sense to say that $p$ and $q$ together relevantly CL-imply $p$, as $q$ is completely irrelevant for the entailment. Although $p$ relevantly CL-implies $p, p$ and $q$ together do not.

Let us take a look at another example. It is unproblematic to claim that $p$ relevantly CL-implies $p \vee q$. It is also clear that $\neg p$ and $p \vee q$ together relevantly CL-imply $q$. It is however not the case that $p$ and $\neg p$ together relevantly CL-imply $q$. While in this case both of the premises are relevant, the conclusion is here completely arbitrary, i.e. $q$ could be replaced by whatever formula.

preservation.

Australasian Journal of Logic (16:2) 2019 Article no. 1 
It can be seen from these examples that, whereas CL-consequence is of course reflexive, monotonic and transitive, relevant $\mathbf{C L}$-implication does not always satisfy all of these properties (but remark that we do have reflexivity: $A$ relevantly implies $A$, in every logic).

Given that we require every premise of a relevant validity to be nonredundant in obtaining the conclusion, it comes as no surprise that our notion is non-monotonic (in the same sense in which also logics in the relevance logic tradition are non-monotonic - for the precise relation with traditional relevance logic, see below). But it may be more surprising that our relevant validity relation is not transitive. Many relevance logicians may even strongly object against the lack of transitivity. It is of course true that, in order to be able to formalize standard deductive practices, we need some kind of a notion of 'implication' which is (at least cautiously ${ }^{3}$ ) transitive. Otherwise one cannot allow for cumulative theorem proving: it is an established practice that once one has proven a certain theorem from axioms, one can use this theorem in further derivations as if it were an axiom, without further proof. So, to formalize mathematical and scientific theories, we definitely need a notion of consequence relation which is transitive as the underlying logic of such theories. But this does not mean that the notion of relevant validity has to be transitive. There is no fundamental reason why the notion 'relevant validity' should coincide with the notion of consequence that underlies our theories. Just like it is generally accepted and unproblematic that there is non-transitivity in counterfactual and indicative conditionals, also relevant validity may be non-transitive, without this needing to affect the logical structure of our theories.

Many relevance logicians have aimed to come up with a holistic alternative to classical logic (this is clear in the philosophical project presented in, for example, [2] and [14]). Given that they entirely reject classical logic, they had to come up with alternative ways to formalize the notion of truth preservation, consequence, the underlying logic of theories, etc. Here we only reject the claim that $\mathbf{C L}$-consequence would be a good characterization of relevant validity. We do not reject other uses of this logic.

Transitivity is a far from obvious property of relevant implication. Consider that we can only say that $A$ relevantly implies $B$ if there is a connection between $A$ and $B$. If we know that $A$ relevantly implies $B$ and $B$ relevantly implies $C$ we sure know that $A$ implies $C$ and that there is a link between

\footnotetext{
${ }^{3}$ A relation $\vdash$ is cautiously transitive iff, whenever $\Gamma \vdash A$ and $\Gamma \cup\{A\} \vdash B$, then $\Gamma \vdash B$.
} 
both $A$ and $B$ and between $B$ and $C$. But we have no information about there being a link between $A$ and $C$. So it would be very strange that, without further information, it would always be true that $A$ relevantly implies $C$. Why then would we even expect relevant implication to be transitive? Might it not be so that one has always accepted transitivity for relevant implication (without convincing argument) only because one was so used to it from more traditional logics?

Note that, when there is exactly one premise and one conclusion, our relevant CL-implication coincides with Smiley's alternative concept of logical consequence (see [17, Section 2]) and Burgess's 'perfectible' entailment relation (see [5]). Burgess begins by stipulating that $A$ perfectly entails $B$ iff $A \vdash_{\mathbf{C L}} B, \nvdash_{\mathbf{C L}} B$ and $\nvdash_{\mathbf{C L}} \neg A$ and goes on to define that $A$ perfectibly entails $B$ iff there is an abstraction $A^{\prime}, B^{\prime}$ of $A, B$ such that $A^{\prime}$ perfectly entails $B^{\prime}$. It is clear that $A$ relevantly $\mathbf{C L}$-implies $B$ (in our sense) iff $A$ perfectibly entails $B$. However in case we have zero or more than one premises or conclusions (or a different logic), Burgess's definition cannot be used.

Although we now have given a precise definition of the relevant CLimplication relation, we have not yet provided a complete logical formalization of the relevant implication connective. We have not yet presented how to prove that something relevantly $\mathbf{C L}$-implies something else. Where $\boldsymbol{\Gamma}$ and $\boldsymbol{\Delta}$ are classical logic formulas, define $\vDash_{\mathbf{N T R}}$ by $\boldsymbol{\Gamma} \vDash_{\mathbf{N T R}} \boldsymbol{\Delta}$ iff $\boldsymbol{\Gamma}$ relevantly $\mathbf{C L}$ imply $\boldsymbol{\Delta}$. In the next section we will present a sequent calculus for $\vDash_{\text {NTR }}$ and prove that it is sound and complete.

By means of this formalization of relevant CL-implication alone we do not yet have presented how the relevant implication relation can be nested and how it can be related to the usual object language logical vocabulary.

In the relevance logic tradition of Routley, Meyer, Anderson, Belnap, Dunn and many others (see [2], [3], [9], [8], [13], [14], [16], [15], and [21]), one gives a formal definition of relevant implication by adding the relevant implication to the object language in the form of an arrow which can be used on the same level as what is usually seen as logical vocabulary (conjunction, disjunction, negation, equivalence, etc.). In the third section of this paper, we do the same thing, resulting in the logic $\mathbf{N T R} \rightarrow$. NTR $\rightarrow$ will prove $A_{1} \rightarrow$ $\left(A_{2} \rightarrow \ldots \rightarrow\left(A_{n} \rightarrow B\right) \ldots\right)$ (the last "..." contains only parentheses) iff $A_{1}$, $A_{2}, \ldots$, and $A_{n}$ together relevantly CL-imply $B$. For more complex nested implications our relevant implication will function much like the relevant implication of $\mathbf{R}$.

Australasian Journal of Logic (16:2) 2019 Article no. 1 


\section{Relevant CL-implication: a sequent calcu- lus for NTR}

We start by defining the (multiset) sequent calculus for NTR.

\subsection{Definition}

Definition 4. Syntactic consequence. Where $\boldsymbol{\Gamma}$ and $\boldsymbol{\Delta}$ are multisets of propositional formulas the only logical symbols in which are $\vee$ and $\neg, \boldsymbol{\Gamma} \vdash_{\mathbf{N T R}} \boldsymbol{\Delta}$ iff the sequent $\boldsymbol{\Gamma} \triangleright \boldsymbol{\Delta}$ is derivable by means of the rules and axioms listed below.

The only axiom schema:

$$
A \triangleright A
$$

The only structural rule:

$$
\frac{\boldsymbol{\Gamma}, A, A \triangleright \boldsymbol{\Delta}}{\boldsymbol{\Gamma}, A \triangleright \boldsymbol{\Delta}} \quad \frac{\boldsymbol{\Gamma} \triangleright A, A, \boldsymbol{\Delta}}{\boldsymbol{\Gamma} \triangleright A, \boldsymbol{B C O N}}
$$

The rules for $\neg$ :

$$
\left.\left.\frac{\Gamma, A \triangleright \boldsymbol{\Delta}}{\Gamma \triangleright \neg A, \boldsymbol{\Delta}}\right\urcorner \frac{\boldsymbol{\Gamma} \triangleright A, \boldsymbol{\Delta}}{\boldsymbol{\Delta}, \neg A \triangleright \boldsymbol{\Delta}} \mathrm{L}\right\urcorner
$$

The rules for $\vee$ :

$$
\frac{\boldsymbol{\Gamma} \triangleright A, \boldsymbol{\Delta}}{\boldsymbol{\Gamma} \triangleright A \vee B, \boldsymbol{\Delta}} \mathrm{R \vee 1} \quad \frac{\boldsymbol{\Gamma} \triangleright B, \boldsymbol{\Delta}}{\boldsymbol{\Gamma} \triangleright A \vee B, \boldsymbol{\Delta}} \mathrm{R \vee 2} \quad \frac{\boldsymbol{\Gamma}_{\mathbf{1}}, A \triangleright \boldsymbol{\Delta}_{\mathbf{1}} \quad \boldsymbol{\Gamma}_{\mathbf{2}}, B \triangleright \boldsymbol{\Delta}_{2}}{\boldsymbol{\Gamma}_{1}, \boldsymbol{\Gamma}_{2}, A \vee B \triangleright \boldsymbol{\Delta}_{1}, \boldsymbol{\Delta}_{2}} \mathrm{LV}
$$

\subsection{Derived rules}

We present some useful derived rules, the first two derivable by means of the corresponding primitive rules plus applications of the LCON and RCON rules, the last is a special case of the second.

$$
\begin{gathered}
\frac{\boldsymbol{\Gamma} \triangleright A, B, \boldsymbol{\Delta}}{\boldsymbol{\Gamma} \triangleright A \vee B, \boldsymbol{\Delta}} \mathrm{R \vee f} \\
\frac{\boldsymbol{\Gamma}_{\mathbf{1}}, A \triangleright \boldsymbol{\Delta}_{\mathbf{1}} \quad \boldsymbol{\Gamma}_{\mathbf{2}}, B \triangleright \boldsymbol{\Delta}_{\mathbf{2}}}{\left(\boldsymbol{\Gamma}_{\mathbf{1}} \cup \boldsymbol{\Gamma}_{\mathbf{2}}\right)-\left(\boldsymbol{\Gamma}_{\mathbf{1}} \cap \boldsymbol{\Gamma}_{\mathbf{2}}\right), \boldsymbol{\Gamma}_{\mathbf{2}}, A \vee B \triangleright\left(\boldsymbol{\Delta}_{\mathbf{1}} \cup \boldsymbol{\Delta}_{\mathbf{2}}\right)-\left(\boldsymbol{\Delta}_{\mathbf{1}} \cap \boldsymbol{\Delta}_{\mathbf{2}}\right)} \mathrm{L \vee c} \\
\frac{\boldsymbol{\Gamma}, A \triangleright \boldsymbol{\Delta} \quad \boldsymbol{\Gamma}, B \triangleright \boldsymbol{\Delta}}{\boldsymbol{\Gamma}, A \vee B \triangleright \boldsymbol{\Delta}} \mathrm{L \vee f}
\end{gathered}
$$

Australasian Journal of Logic (16:2) 2019 Article no. 1 
The difference between $L \vee c$ and $L \vee$ lies in the fact that we are dealing with multisets. If there is a formula $A$ that occurs both in $\boldsymbol{\Gamma}_{\mathbf{1}}$ and $\boldsymbol{\Gamma}_{\mathbf{2}}, \mathrm{LV}$ requires to keep the copies $A$ in $\boldsymbol{\Gamma}_{\mathbf{1}}$ plus those in $\boldsymbol{\Gamma}_{\mathbf{2}}$ while $\mathrm{L} \vee \mathrm{c}$ allows you to only keep the number of copies present in either $\boldsymbol{\Gamma}_{\mathbf{1}}$ or $\boldsymbol{\Gamma}_{\mathbf{2}}$, depending of which has the most $A$ 's. For example, if $\boldsymbol{\Gamma}_{\mathbf{1}}=\{A, A, B\}$ and $\boldsymbol{\Gamma}_{\mathbf{2}}=\{A, A, A, C\}$, then $\left(\boldsymbol{\Gamma}_{\mathbf{1}} \cup \boldsymbol{\Gamma}_{\mathbf{2}}\right)-\left(\boldsymbol{\Gamma}_{\mathbf{1}} \cap \boldsymbol{\Gamma}_{\mathbf{2}}\right)=\{A, A, A, B, C\}$, while $\boldsymbol{\Gamma}_{\mathbf{1}}, \boldsymbol{\Gamma}_{\mathbf{2}}$ or $\boldsymbol{\Gamma}_{\mathbf{1}} \cup \boldsymbol{\Gamma}_{\mathbf{2}}$ is simply $\{A, A, A, A, A, B, C\}$.

We can define the other traditional logical symbols, as follows:

$$
\begin{aligned}
& A \wedge B={ }_{d f} \neg(\neg A \vee \neg B) \\
& A \supset B=_{d f} \neg A \vee B \\
& A \equiv B=_{d f}(A \supset B) \wedge(B \subset A)
\end{aligned}
$$

Now one can easily derive introduction rules for these defined symbols.

$$
\begin{aligned}
& \frac{\boldsymbol{\Gamma}, A \triangleright \boldsymbol{\Delta}}{\boldsymbol{\Gamma} \triangleright A \supset B, \boldsymbol{\Delta}} \mathrm{R \supset 1} \quad \frac{\boldsymbol{\Gamma} \triangleright B, \boldsymbol{\Delta}}{\boldsymbol{\Gamma} \triangleright A \supset B, \boldsymbol{\Delta}} \mathrm{R \supset 2} \quad \frac{\boldsymbol{\Gamma}_{\mathbf{1}} \triangleright A, \boldsymbol{\Delta}_{\mathbf{1}} \quad \boldsymbol{\Gamma}_{\mathbf{2}}, B \triangleright \boldsymbol{\Delta}_{2}}{\boldsymbol{\Gamma}_{1}, \boldsymbol{\Gamma}_{2}, A \supset B \triangleright \boldsymbol{\Delta}_{1}, \boldsymbol{\Delta}_{2}} \mathrm{~L} \\
& \frac{\boldsymbol{\Gamma}_{\mathbf{1}} \triangleright A, \boldsymbol{\Delta}_{\mathbf{1}} \quad \boldsymbol{\Gamma}_{\mathbf{2}} \triangleright B, \boldsymbol{\Delta}_{2}}{\boldsymbol{\Gamma}_{1}, \boldsymbol{\Gamma}_{2} \triangleright A \wedge B, \boldsymbol{\Delta}_{1}, \boldsymbol{\Delta}_{2}} \mathrm{R} \wedge \quad \frac{\boldsymbol{\Gamma}, A \triangleright \boldsymbol{\Delta}}{\boldsymbol{\Gamma}, A \wedge B \triangleright \boldsymbol{\Delta}} \mathrm{L} \wedge 1 \quad \frac{\boldsymbol{\Gamma}, B \triangleright \boldsymbol{\Delta}}{\boldsymbol{\Gamma}, A \wedge B \triangleright \boldsymbol{\Delta}} \mathrm{L} \wedge 2 \\
& \frac{\boldsymbol{\Gamma}_{\mathbf{1}} \triangleright A, \boldsymbol{\Delta}_{\mathbf{1}} \quad \boldsymbol{\Gamma}_{\mathbf{2}}, B \triangleright \boldsymbol{\Delta}_{2}}{\boldsymbol{\Gamma}_{1}, \boldsymbol{\Gamma}_{2}, A \equiv B \triangleright \boldsymbol{\Delta}_{1}, \boldsymbol{\Delta}_{2}} \mathrm{~L} \equiv 1 \quad \frac{\boldsymbol{\Gamma}_{\mathbf{1}}, A \triangleright \boldsymbol{\Delta}_{\mathbf{1}} \quad \boldsymbol{\Gamma}_{\mathbf{2}} \triangleright B, \boldsymbol{\Delta}_{2}}{\boldsymbol{\Gamma}_{1}, \boldsymbol{\Gamma}_{2}, A \equiv B \triangleright \boldsymbol{\Delta}_{1}, \boldsymbol{\Delta}_{2}} \mathrm{~L} \equiv 2 \\
& \frac{\boldsymbol{\Gamma}_{\mathbf{1}} \triangleright A, \boldsymbol{\Delta}_{\mathbf{1}} \quad \boldsymbol{\Gamma}_{\mathbf{2}} \triangleright B, \boldsymbol{\Delta}_{2}}{\boldsymbol{\Gamma}_{1}, \boldsymbol{\Gamma}_{2} \triangleright A \equiv B, \boldsymbol{\Delta}_{1}, \boldsymbol{\Delta}_{2}} \mathrm{R} \equiv 1 \quad \frac{\boldsymbol{\Gamma}_{\mathbf{1}}, A \triangleright \boldsymbol{\Delta}_{\mathbf{1}} \quad \boldsymbol{\Gamma}_{\mathbf{2}}, B \triangleright \boldsymbol{\Delta}_{2}}{\boldsymbol{\Gamma}_{1}, \boldsymbol{\Gamma}_{2} \triangleright A \equiv B, \boldsymbol{\Delta}_{1}, \boldsymbol{\Delta}_{2}} \mathrm{R} \equiv 2
\end{aligned}
$$

We conclude with rules to conjoin premises and disjoin conclusions.

$$
\frac{\Gamma_{1}, \Gamma_{2} \triangleright \Delta}{\Gamma, \bigwedge \Gamma_{2} \triangleright \Delta} \mathrm{L} \wedge \mathrm{m} \quad \frac{\Gamma \triangleright \Delta_{1}, \Delta_{2}}{\Gamma \triangleright \bigvee \Delta_{1}, \Delta_{2}} \mathrm{R} \vee \mathrm{m}
$$

\subsection{Examples}

NTR-proof for $r \wedge q \triangleright \neg p \vee(p \wedge q)$

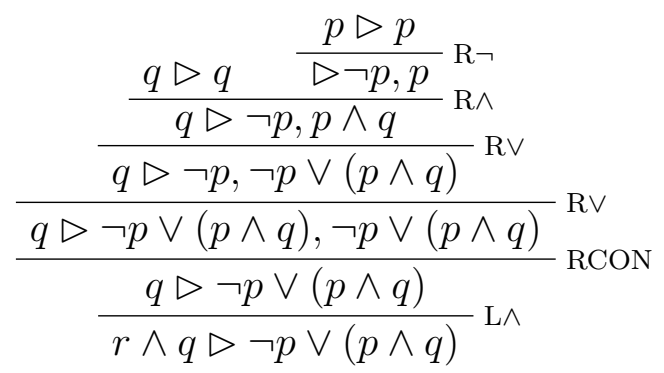

Australasian Journal of Logic (16:2) 2019 Article no. 1 
NTR-proof for $(p \wedge \neg p) \vee q \triangleright q \wedge(\neg r \vee r)$

$$
\begin{aligned}
& \frac{p \triangleright p}{\neg p, p \triangleright} \mathrm{L}-
\end{aligned}
$$

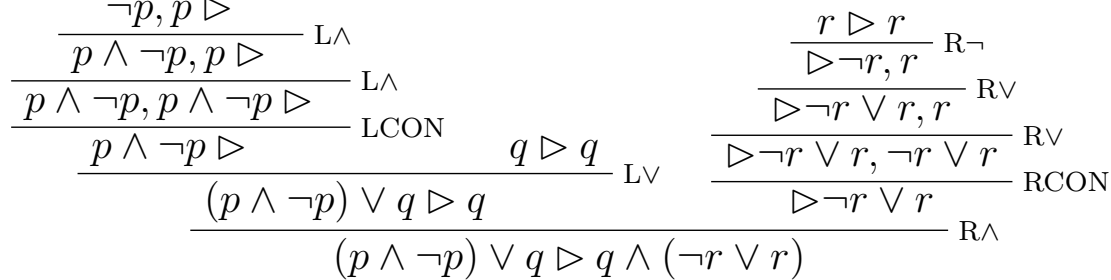

NTR-proof for $p \wedge p \triangleright p, p \wedge \neg p$

$$
\frac{p \triangleright p \quad \frac{p \triangleright p}{\triangleright \neg p, p}}{\frac{\mathrm{R} \neg}{p \triangleright p, p \wedge \neg p}} \mathrm{R} \wedge
$$

NTR-proof for $(p \vee r) \wedge(p \vee s) \wedge(q \vee r) \wedge(q \vee s) \triangleright(p \wedge q) \vee(r \wedge s)$

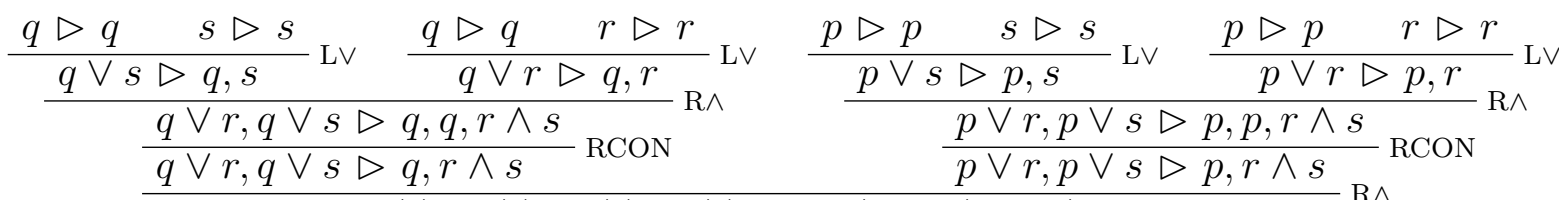

$$
\begin{aligned}
& \frac{p \vee r, p \vee s, q \vee r, q \vee s \triangleright p \wedge q, r \wedge s, r \wedge s}{p \vee r, p \vee s, q \vee r, q \vee s \triangleright p \wedge q, r \wedge s} \mathrm{RCON} \\
& p \vee r, p \vee s, q \vee r, q \vee s \triangleright(p \wedge q) \vee(r \wedge s)-\mathrm{R} \vee \mathrm{m}
\end{aligned}
$$

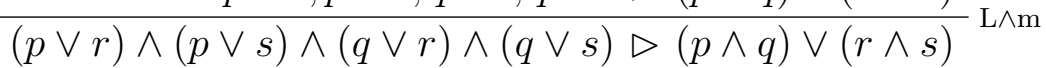

\subsection{Soundness}

Theorem 1. Soundness. If $\boldsymbol{\Gamma}^{\mathrm{s}} \vdash_{\mathrm{NTR}} \boldsymbol{\Delta}^{\mathrm{s}}$, then ${ }^{4} \boldsymbol{\Gamma}^{\mathrm{s}} \models_{\mathrm{NTR}} \boldsymbol{\Delta}^{\mathbf{s}}$.

Proof. We need to prove that, for every NTR-proof, the final conclusion always has a CL-valid abstraction such that no proper subsequent is CLvalid. We do this recursively.

\footnotetext{
${ }^{4}$ The superscripts $s$ and $a$ in $\Gamma^{\mathbf{s}}$ and $\Gamma^{\mathbf{a}}$ have no meaning, they just indicate different metavariables.
}

Australasian Journal of Logic (16:2) 2019 Article no. 1 
This holds obviously for every NTR-proof only existing of an axiom. The only sequent in such a proof can always be abstracted into the CLvalid sequent $p \triangleright p$. The reader sees that all its proper subsequents are not CL-valid.

We need to show for each rule that, if its local premises ${ }^{5}$ have a CL-valid abstraction such that none of its proper subsequents are CL-valid, then there is also such a non-redundant abstraction of the local conclusion of the rule. We treat the rules one by one. Each time we suppose there is such a nonredundant abstraction for the local premises.

1. LV. Transform the non-redundant abstraction of the local premises by relettering (substitute letters by other letters) in such a way that the transformed abstractions of the two local premises have no letters in common. Say the transformed abstractions (which are also nonredundant abstractions!) are $\boldsymbol{\Gamma}_{\mathbf{1}}, A_{1}, A_{2} \ldots A_{n} \triangleright \boldsymbol{\Delta}_{\mathbf{1}}$ and $\boldsymbol{\Gamma}_{\mathbf{2}}, B_{1}, B_{2} \ldots B_{m} \triangleright$ $\boldsymbol{\Delta}_{\mathbf{2}}$, where $A_{1}, A_{2} \ldots A_{n}$ and $B_{1}, B_{2} \ldots B_{m}$ are the abstractions of resp. $A$ and $B$. The sequent $\boldsymbol{\Gamma}_{\mathbf{1}}, \boldsymbol{\Gamma}_{\mathbf{2}}, A_{1} \vee B_{1}, A_{1} \vee B_{2}, \ldots A_{1} \vee B_{m}, \ldots A_{n} \vee$ $B_{1}, A_{1} \vee B_{2}, \ldots A_{n} \vee B_{m} \triangleright \boldsymbol{\Delta}_{\mathbf{1}}, \boldsymbol{\Delta}_{\mathbf{2}}$ is an abstraction of the local conclusion of $\mathrm{L} \vee$, is $\mathbf{C L}$-valid and it cannot have a proper subsequent that is $\mathbf{C L}$-valid because $\boldsymbol{\Gamma}_{\mathbf{1}}, A_{1}, A_{2} \ldots A_{n} \triangleright \boldsymbol{\Delta}_{\mathbf{1}}$ and $\boldsymbol{\Gamma}_{\mathbf{2}}, B_{1}, B_{2} \ldots B_{m} \triangleright \boldsymbol{\Delta}_{\mathbf{2}}$ are non-redundant and have no letters in common.

2. $\mathrm{R} \vee 1$. Let $\boldsymbol{\Gamma} \triangleright A_{1}, \ldots A_{n}, \Delta$ be the non-redundant abstraction of the local premise, where $A_{1}, A_{2} \ldots A_{n}$ is the abstraction of $A$. Take as the non-redundant abstraction of the local conclusion $\Gamma \triangleright A_{1} \vee \sigma, A_{2} \vee$ $\sigma, \ldots A_{n} \vee \sigma, \Delta$, where $\sigma$ is a letter that does not occur in the abstraction of the local premise.

3. $\mathrm{R} \vee 2$. Similar to $R \vee 1$.

4. $\mathrm{R} \neg$ and $\mathrm{L} \neg$. Evident in view of the fact that the local premise is CLvalid iff the local conclusion is. Take as the non-redundant abstraction of the conclusion the abstraction of the local premise such that $A_{1}, A_{2} \ldots A_{n}$ is removed and $\neg A_{1}, \neg A_{2} \ldots \neg A_{n}$ is added on the other side of $\triangleright$, where $A_{1}, A_{2} \ldots A_{n}$ is the abstraction of the formula that is negated by the rule.

\footnotetext{
${ }^{5}$ The local premises of a rule are the sequents that are used by the rule to obtain a new sequent (the local conclusion). In other words, local premises are the sequents above and the local conclusion the sequent below the line in the definition of the rule.
}

Australasian Journal of Logic (16:2) 2019 Article no. 1 
5. LCON and RCON. The non-redundant abstraction of the local premise is also a non-redundant abstraction of the local conclusion.

\subsection{Completeness}

Theorem 2. Completeness. If $\boldsymbol{\Gamma}^{\mathrm{s}} \vDash_{\mathbf{N T R}} \boldsymbol{\Delta}^{\mathrm{s}}$, then $\boldsymbol{\Gamma}^{\mathbf{s}} \vdash_{\mathbf{N T R}} \boldsymbol{\Delta}^{\mathbf{s}}$.

Before moving to the actual proof, we prepare the proof with some useful terminology. Let an NTR-tree be a tree of sequents that respects all rules of NTR, but in which the leafs are not necessarily axioms. We say that an NTR-tree is an NTR-tree for a sequent if that sequent is the root of the tree. An NTR-tree is tableau-like iff each rule used in the tree is one of $\mathrm{L} \vee \mathrm{f}$, $\mathrm{R} \vee \mathrm{f}, \mathrm{L} \neg$, or $\mathrm{R} \neg$.

An NTR-tree is completed iff all formulas that occur in the leafs are atoms. Note that there is at least one tableau-like completed NTR-tree for each sequent, as we can always further analyse every remaining complex formula by one of the rules $L \vee f, R \vee f, L \neg$, or $R \neg$.

An NTR-tree is a proto-proof iff all its leafs are sequents such that at least one formula occurs both left and right of $\triangleright$. The reader can easily verify that, whenever there is a proto-proof for a sequent, then that sequent is CL-valid.

Successor and predecessor (recursive definition). An occurrence $O_{1}$ of a formula in a sequent $S_{1}$ is a successor of an occurrence $O_{2}$ of a formula in another sequent $S_{2}$ iff (i) $S_{1}$ is the local conclusion of an application of a rule with $S_{2}$ as a local premise, $O_{1}$ and $O_{2}$ encode the same formula, and $O_{1}$ and $\mathrm{O}_{2}$ are in the part of the sequent that is left untouched by the rule, (ii) $S_{1}$ is the local conclusion of an application of a rule with $S_{2}$ as a local premise and the $O_{1}$ is the result of the application of the rule on $O_{2}$, or (iii) $O_{1}$ is a successor of another occurrence $O_{3}$ in another sequent and $O_{3}$ is a successor of $O_{2}$. $O_{1}$ is a predecessor of $O_{2}$ iff $O_{2}$ is the successor of $O_{1}$.

Proof. Suppose $\boldsymbol{\Gamma}^{\mathbf{s}} \nvdash_{\mathbf{N T R}} \boldsymbol{\Delta}^{\mathbf{s}}$. We will show that $\boldsymbol{\Gamma}^{\mathbf{s}} \not \nvdash_{\mathbf{N T R}} \boldsymbol{\Delta}^{\mathbf{s}}$.

Maximal analysis Given that there is no $\mathbf{N T R}$-proof for $\boldsymbol{\Gamma}^{\mathbf{s}} \triangleright \boldsymbol{\Delta}^{\mathbf{s}}$, all the completed trees for $\Gamma^{\mathbf{s}} \triangleright_{\mathbf{N T R}} \boldsymbol{\Delta}^{\mathbf{s}}$ have at least one leaf that is not $\sigma \triangleright \sigma$.

Australasian Journal of Logic (16:2) 2019 Article no. 1 
Take any tableau-like completed tree $T$. There are two possibilities: either there is a tableau-like completed tree with a leaf containing $\Gamma^{\prime} \nabla^{\boldsymbol{\Delta}^{\prime}}$ s.t. $\Gamma^{\prime} \cap \boldsymbol{\Delta}^{\prime}=\emptyset$, or there is no such tree.

Not a classical consequence In the case there is such a tree with a leaf containing $\boldsymbol{\Gamma}^{\prime} \triangleright \boldsymbol{\Delta}^{\prime}$ s.t. $\boldsymbol{\Gamma}^{\prime} \cap \boldsymbol{\Delta}^{\prime}=\emptyset$, the contents of that leaf constitute a countermodel for $\Gamma \vdash_{\mathbf{C L}} \Delta$ (let the letters in $\boldsymbol{\Gamma}^{\prime}$ be true and the ones in $\boldsymbol{\Delta}^{\prime}$ be false). This can be shown recursively by demonstrating that every sequent on a branch in a tableau-like completed tree with such a leaf is CL-falsified by each model that makes all atoms in $\boldsymbol{\Gamma}^{\prime}$ true and all atoms in $\boldsymbol{\Delta}^{\prime}$ false. Of course the final conclusion of the tableau-like tree is then also CL-falsified by each such model.

Irrelevant consequence If there is no tree with such a countermodel leaf, all leafs of all completed trees are such that there is an atom that occurs both left and right of $\triangleright$. In that case the tree is a proto-proof and so the final conclusion is CL-valid. Now we need to prove that it is however not NTRvalid.

In order to do that, we need to show that every CL-valid abstraction of $\boldsymbol{\Gamma} \triangleright \boldsymbol{\Delta}$ has a proper subsequent that is also CL-valid. Let $\boldsymbol{\Gamma}^{\mathbf{a}} \nabla \boldsymbol{\Delta}^{\mathbf{a}}$ be an arbitrary CL-valid abstraction. From the fact that there is no NTRproof for $\boldsymbol{\Gamma}^{\mathbf{s}} \triangleright \boldsymbol{\Delta}^{\mathbf{s}}$ we can conclude that there is none for $\boldsymbol{\Gamma}^{\mathbf{a}} \nabla \boldsymbol{\Delta}^{\mathbf{a}}$ either (all NTR-rules are formal; a uniform substitution of a proof will also constitute a correct $\mathbf{N T R}$-proof). We will construct a proto-proof for $\boldsymbol{\Gamma}^{\mathbf{a}} \triangleright \boldsymbol{\Delta}^{\mathbf{a}}$ with the special property that if one leaf of that tree contains a sequent that does not comply with the schema for NTR-axioms, then a proper subsequent of the final conclusion of the tree is also CL-valid. Because there is no NTRproof, there cannot be a proto-proof tree in which all leafs comply with that schema. We can conclude that, by means of that proto-proof construction, we will have proven the redundancy of each abstraction of $\boldsymbol{\Gamma}^{\mathbf{s}} \Delta \boldsymbol{\Delta}^{\mathbf{s}}$. We will be able to conclude that $\Gamma^{\mathbf{s}} \triangleright \boldsymbol{\Delta}^{\mathbf{s}}$ is not $\mathbf{N} \mathbf{T R}$-valid.

Stage 1. First, consider that, since $\Gamma^{\mathbf{a}} \triangleright \boldsymbol{\Delta}^{\mathbf{a}}$ is $\mathbf{C L}$-valid, there exists a tableau-like proto-proof $T_{1}$ for it in view of the completeness of proto-proofs w.r.t. classical logic (which can be proven using exactly the same methods used to prove the completeness of traditional tableaux methods).

Stage 2. Then we construct a more parsimonious proto-proof for $\Gamma^{\mathbf{a}} \triangleright \boldsymbol{\Delta}^{\mathbf{a}}$ by removing all redundancies from $T_{1}$, as follows. Let a pruning of a proto-

Australasian Journal of Logic (16:2) 2019 Article no. 1 
proof be a tree that is the result of removing a formula and all its predecessors from the proto-proof in such a way that the resulting tree is also a protoproof (after one replacing at most one time $\mathrm{R} \vee f b^{6} \mathrm{R} \vee c$ and possibly making several rule applications empty, i.e. the local premise is exactly the same as the local conclusion). Now maximally prune $T_{1}$ (i.e. there is a sequence $\left\langle T_{1}, T_{b 2}, \ldots, T_{b n-1}, T_{b n}\right\rangle$ s.t. $T_{b 2}=$ a pruning of $T_{1}, T_{b 3}$ is a pruning of $T b_{2}, \ldots$, $T_{b n}$ is a pruning of $T_{b n-1}$, and $T_{b n}$ does not have a pruning) resulting in $T_{2}$.

Let us give an example of this pruning process.

$$
\frac{\left.\frac{q, p \triangleright p, r, s}{q, p, \neg p \triangleright r, s} \mathrm{~L}\right\urcorner \quad q, p, r \triangleright r, s}{\frac{q, p, \neg p \vee r \triangleright r, s}{q, p, \neg p \vee r \triangleright r \vee s} \mathrm{R \vee f}} \mathrm{L \vee f}
$$

First pruning

$$
\frac{\left.\frac{q, p \triangleright p, r}{q, p, \neg p \triangleright r} \mathrm{~L}\right\urcorner \quad q, p, r \triangleright r}{\frac{q, p, \neg p \vee r \triangleright r}{q, p, \neg p \vee r \triangleright r \vee s} \mathrm{R} \vee} \mathrm{L} \mathrm{f}
$$

Second pruning

$$
\frac{\left.\frac{p \triangleright p, r}{p, \neg p \triangleright r} \mathrm{~L}\right\urcorner \quad q, p, r \triangleright r}{\frac{q, p, \neg p \vee r \triangleright r}{q, p, \neg p \vee r \triangleright r \vee s} \mathrm{R} \vee} \mathrm{L} \vee
$$

Third pruning

$$
\frac{\left.\frac{p \triangleright p, r}{p, \neg p \triangleright r} \mathrm{~L}\right\urcorner \quad q, r \triangleright r}{\frac{q, p, \neg p \vee r \triangleright r}{q, p, \neg p \vee r \triangleright r \vee s} \mathrm{R} \vee} \mathrm{L}
$$

Fourth pruning

$$
\frac{\left.\frac{p \triangleright p}{p, \neg p \triangleright} \mathrm{L}\right\urcorner \quad q, r \triangleright r}{\frac{q, p, \neg p \vee r \triangleright r}{q, p, \neg p \vee r \triangleright r \vee s} \mathrm{~L} \vee}
$$

\footnotetext{
${ }^{6}$ If one removes an occurrence of a formula from sequents in a proof, it will no longer be a correct application of the same rule, so one needs to change the justification of the step in the proof.
}

Australasian Journal of Logic (16:2) 2019 Article no. 1 
Every further removal of formulas from the tree results in a violation of rules or another final conclusion sequent.

Stage 3. We recursively construct a specific proto-proof $T_{3}$ from $T_{2}$ by removing formulas and subtrees from $T_{3}$ such that the final conclusion of $T_{3}$ is a proper subsequent of the final conclusion of $T_{2}$. Because $T_{3}$ is a protoproof, its final conclusion is CL-valid, and so the final conclusion of $T_{2}$ has a CL-valid subsequent.

We construct $T_{3}$ following the same tree structure as $T_{2}$, sequent per sequent each time mentioning which formulas or even whole subtrees need to be removed from $T_{2}$ in order to obtain $T_{3}$.

First we construct the leafs of $T_{3}$ based on those of $T_{2}$. At least one leaf sequent in $T_{2}$ contains a formula that may be removed so that the leaf would still be a leaf of a proto-proof (such as the sequent $q, r \triangleright r$ in one of the example tree in Stage 2, in this sequent $q$ can be removed; $r \triangleright r$ is still an acceptable leaf of a proto-proof), otherwise the tree would be an NTR-proof. Remove this redundant formula from such a leaf in $T_{2}$ to obtain the corresponding leaf in $T_{3}$. The other leafs of $T_{3}$ are the same as the corresponding leafs in $T_{2}$. Call the leaf that differs the slimmed down leaf.

Now we assume already to have constructed $T_{3}$ upto a certain point. Consider arbitrary subtrees $T_{2}^{\prime a}$ and $T_{2}^{\prime b}$ of $T_{2}$. Suppose we already have the corresponding trees $T_{3}^{\prime a}$ and $T_{3}^{\prime b}$ which are proto-proofs and for which the final conclusion is a proper subsequent of the final conclusion of corresponding tree $T_{2}^{\prime x}$, whenever the slimmed down leaf is in that subtree $T_{3}^{\prime}$, and $T_{3}^{\prime x}=T_{2}^{\prime x}$ otherwise. We prove that the subproof $T_{2}^{\prime \prime}$ of $T_{2}$ that is result of applying a rule $R$ to $T_{2}^{\prime a}$ and $T_{2}^{\prime b}$ (or one of them, in case of a rule with only one local premise) can also be transformed into $T_{3}^{\prime \prime}$ (it is a proto-proof and has as final conclusion a proper subsequent of $T_{2}^{\prime \prime \prime}$ 's final conclusion, whenever the slimmed down leaf is inside there).

Let $T_{3}^{\prime \prime}$ be identical to $T_{2}^{\prime \prime}$ if the slimmed down leaf is not inside of $T_{3}^{\prime a}$ nor inside of $T_{3}^{\prime b}$.

Otherwise call $T_{3}^{\prime}$ the tree with the slimmed down leaf, and $T_{3}^{\prime b}$ the other one; $T_{2}^{\prime}$ and $T_{2}^{\prime b}$ are the corresponding subtrees of $T_{2}$. If the rule $R$ has only one local premise, it suffices to speak of $T_{3}^{\prime}$ and $T_{2}^{\prime}$ and drop the $a$ and $b$ altogether.

We construct $T_{3}^{\prime \prime}$ depending on the rule $R$ used to construct $T_{2}^{\prime \prime}$, assuming that $T_{3}^{\prime}$ contains the slimmed down leaf and has as its final conclusion a proper subsequent of the $T_{2}^{\prime \prime}$ s final conclusion. The final conclusion of $T_{3}^{\prime b}$ is always identical to that of $T_{2}^{\prime b}$. We need to treat the rules that may occur in 
$T_{2}^{\prime}$ case by case.

1. L $\vee$ c. Let the final conclusion of $T_{2}^{\prime}$ be $\boldsymbol{\Gamma}_{\mathbf{1}}, A \triangleright \boldsymbol{\Delta}_{\mathbf{1}}$, the final conclusion of both $T_{b}^{\prime 3}$ and $T_{b}^{\prime 2}$ is $\boldsymbol{\Gamma}_{\mathbf{2}}, B \triangleright \boldsymbol{\Delta}_{\mathbf{2}}$, and the final conclusion of $T_{2}^{\prime \prime}$ be $\boldsymbol{\Gamma}_{\mathbf{1}}, \boldsymbol{\Gamma}_{\mathbf{2}}, A \vee B \triangleright \boldsymbol{\Delta}_{\mathbf{1}}, \boldsymbol{\Delta}_{\mathbf{2}}$. There are 3 cases: (1) the final conclusion of $T_{3}^{\prime}$ is of the form $\boldsymbol{\Gamma}_{\mathbf{1}}^{\prime} \triangleright \boldsymbol{\Delta}_{\mathbf{1}}^{\prime}$ where $\boldsymbol{\Gamma}_{\mathbf{1}}^{\prime} \subseteq \boldsymbol{\Gamma}_{\mathbf{1}}$ and $\boldsymbol{\Delta}_{\mathbf{1}}^{\prime} \subseteq \boldsymbol{\Delta}_{\mathbf{1}}$. Let $T_{3}^{\prime \prime}$ be identical to $T_{3}^{\prime}$, because the final conclusion of the latter is already a proper subsequent of the final conclusion of $T_{2}^{\prime \prime}$. (2) The final conclusion of $T_{3}^{\prime}$, say $\boldsymbol{\Gamma}_{\mathbf{1}}^{\prime}, A \triangleright \boldsymbol{\Delta}_{\mathbf{1}}^{\prime}$ is such that $\boldsymbol{\Gamma}_{\mathbf{1}}-\boldsymbol{\Gamma}_{\mathbf{1}}^{\prime} \neq \emptyset$. Then apply $\mathrm{L} \vee \mathrm{c}$ to $T_{3}^{\prime}$ to obtain $T_{3}^{\prime \prime}$, the final conclusion of which is $\left(\boldsymbol{\Gamma}_{\mathbf{1}}^{\prime} \cup \boldsymbol{\Gamma}_{\mathbf{2}}\right)-\left(\boldsymbol{\Gamma}_{\mathbf{1}}^{\prime} \cap \boldsymbol{\Gamma}_{\mathbf{2}}\right), \boldsymbol{\Gamma}_{\mathbf{2}}, A \vee$ $B \triangleright\left(\boldsymbol{\Delta}_{\mathbf{1}}^{\prime} \cup \boldsymbol{\Delta}_{\mathbf{2}}\right)-\left(\boldsymbol{\Delta}_{\mathbf{1}}^{\prime} \cap \boldsymbol{\Delta}_{\mathbf{2}}\right)$. In that case either (1a) $\left(\boldsymbol{\Gamma}_{\mathbf{1}}-\boldsymbol{\Gamma}_{\mathbf{1}}^{\prime}\right) \subseteq \boldsymbol{\Gamma}_{\mathbf{2}}$. This is impossible because, as they are redundant, $T_{2}^{\prime \prime}$ would have been pruned in such a way that all $C \in \boldsymbol{\Gamma}_{\mathbf{1}}-\boldsymbol{\Gamma}_{\mathbf{1}}^{\prime}$ and their predecessors would have been removed from the subproof $T_{2}^{\prime}$ of the proof (remember that $T_{2}$ and all its subproofs are maximally pruned) (1b) otherwise $\left(\boldsymbol{\Gamma}_{\mathbf{1}}^{\prime} \cup \boldsymbol{\Gamma}_{\mathbf{2}}\right)-\left(\boldsymbol{\Gamma}_{\mathbf{1}}^{\prime} \cap \boldsymbol{\Gamma}_{\mathbf{2}}\right)$ is a proper submultiset of $\left(\boldsymbol{\Gamma}_{\mathbf{1}} \cup \boldsymbol{\Gamma}_{\mathbf{2}}\right)-\left(\boldsymbol{\Gamma}_{\mathbf{1}} \cap \boldsymbol{\Gamma}_{\mathbf{2}}\right)$ and $T_{3}^{\prime \prime}$ has a final conclusion that is a proper subsequent that of $T_{2}^{\prime \prime}$. (3) The final conclusion of $T_{3}^{\prime}$, say $\boldsymbol{\Gamma}_{\mathbf{1}}^{\prime}, A \triangleright \boldsymbol{\Delta}_{\mathbf{1}}^{\prime}$ is such that $\Delta_{1}-\Delta_{1}^{\prime} \neq \emptyset$. Very similar to case (2).

2. $\mathrm{R} \vee 1$. Let the final conclusion of $T_{2}^{\prime}$ be $\boldsymbol{\Gamma} \triangleright A, \boldsymbol{\Delta}$ and the final conclusion of $T_{2}^{\prime \prime}$ be $\boldsymbol{\Gamma} \triangleright A \vee B, \boldsymbol{\Delta}$. There are 3 case: (1) the final conclusion of $T_{3}^{\prime}$ is of the form $\boldsymbol{\Gamma}^{\prime} \nabla \boldsymbol{\Delta}^{\prime}$ where $\boldsymbol{\Gamma}^{\prime} \subseteq \boldsymbol{\Gamma}$ and $\boldsymbol{\Delta}^{\prime} \subseteq \boldsymbol{\Delta}$. Let $T_{3}^{\prime \prime}$ be identical to $T_{3}^{\prime}$, because the final conclusion of the latter is already a proper subsequent of the final conclusion of $T_{2}^{\prime \prime}$. (2) The final conclusion of $T_{3}^{\prime}$, say $\Gamma^{\prime} \triangleright A, \boldsymbol{\Delta}^{\prime}$ is such that $\Gamma-\Gamma^{\prime} \neq \emptyset$. Then apply $\mathrm{R} \vee$ to $T_{3}^{\prime}$ to obtain $T_{3}^{\prime \prime}$, the final conclusion of which is $\left(\boldsymbol{\Gamma}^{\prime} \triangleright A \vee B, \boldsymbol{\Delta}^{\prime}\right.$, which is a proper subsequent of the final conclusion of $T_{2}^{\prime \prime}$. (3) The final conclusion of $T_{3}^{\prime}$, say $\Gamma^{\prime} \triangleright A, \boldsymbol{\Delta}^{\prime}$ is such that $\Delta-\Delta^{\prime} \neq \emptyset$. Very similar to case (2).

3. $R \vee 2$. Very similar to $R \vee 1$.

4. L $\neg$. Very similar to $R \vee 1$.

5. Rᄀ. Very similar to $R \vee 1$.

6. $\mathrm{R} \vee f$. Let the final conclusion of $T_{2}^{\prime}$ be $\boldsymbol{\Gamma} \triangleright A, B, \boldsymbol{\Delta}$ and the final conclusion of $T_{2}^{\prime \prime}$ be $\boldsymbol{\Gamma} \triangleright A \vee B, \boldsymbol{\Delta}$. There are 5 case: (1) the final conclusion of $T_{3}^{\prime}$ is of the form $\boldsymbol{\Gamma}^{\prime} \triangleright \boldsymbol{\Delta}^{\prime}$ where $\boldsymbol{\Gamma}^{\prime} \subseteq \boldsymbol{\Gamma}$ and $\boldsymbol{\Delta}^{\prime} \subseteq \boldsymbol{\Delta}$. Let $T_{3}^{\prime \prime}$ be identical to $T_{3}^{\prime}$, because the final conclusion of the latter is already a 
proper subsequent of the final conclusion of $T_{2}^{\prime \prime}$. (2) the final conclusion of $T_{3}^{\prime}$ is of the form $\boldsymbol{\Gamma}^{\prime} \triangleright A, \boldsymbol{\Delta}^{\prime}$ where $\boldsymbol{\Gamma}^{\prime} \subseteq \boldsymbol{\Gamma}$ and $\boldsymbol{\Delta}^{\prime} \subseteq \boldsymbol{\Delta}$. This is impossible because, as $B$ is redundant, $T_{2}^{\prime \prime}$ would have been pruned in such a way that $B$ and its predecessors would have been removed from the subproof $T_{2}^{\prime}$ of the proof (remember that $T_{2}$ and all its subproofs are maximally pruned). (3) the final conclusion of $T_{3}^{\prime}$ is of the form $\boldsymbol{\Gamma}^{\prime} \triangleright B, \boldsymbol{\Delta}^{\prime}$ where $\boldsymbol{\Gamma}^{\prime} \subseteq \boldsymbol{\Gamma}$ and $\boldsymbol{\Delta}^{\prime} \subseteq \boldsymbol{\Delta}$. Similar to case (2). (4) The final conclusion of $T_{3}^{\prime}$, say $\boldsymbol{\Gamma}^{\prime} \triangleright A, B, \boldsymbol{\Delta}^{\prime}$ is such that $\Gamma-\Gamma^{\prime} \neq \emptyset$. Then apply $\mathrm{R} \vee \mathrm{f}$ to $T_{3}^{\prime}$ to obtain $T_{3}^{\prime \prime}$, the final conclusion of which is $\left(\boldsymbol{\Gamma}^{\prime} \triangleright A \vee B, \boldsymbol{\Delta}^{\prime}\right.$, which is a proper subsequent of the final conclusion of $T_{2}^{\prime \prime}$. (5) The final conclusion of $T_{3}^{\prime}$, say $\boldsymbol{\Gamma}^{\prime} \triangleright A, B, \boldsymbol{\Delta}^{\prime}$ is such that $\Delta-\Delta^{\prime} \neq \emptyset$. Very similar to case (4).

This concludes the construction of a proto-proof $T_{3}$. Its final conclusion will be a proper subset of the final conclusion of $T_{2}$, because the slimmed down leaf will be inside $T_{3}$. Because it is a proto-proof, the final conclusion of $T_{3}$ will be $\mathbf{C L}$-valid. That final conclusion is thus a $\mathbf{C L}$-valid proper subsequent of $\boldsymbol{\Gamma}^{\mathbf{a}} \triangleright \boldsymbol{\Delta}^{\mathbf{a}}$. Given that $\boldsymbol{\Gamma}^{\mathbf{a}} \triangleright \boldsymbol{\Delta}^{\mathbf{a}}$ was an arbitrary $\mathbf{C L}$-valid abstraction of $\Gamma^{\mathbf{s}} \triangleright \boldsymbol{\Delta}^{\mathbf{s}}$, there is a CL-valid proper subsequent for every $\mathbf{C L}$-valid abstraction of $\boldsymbol{\Gamma}^{\mathbf{s}} \triangleright \boldsymbol{\Delta}^{\mathbf{s}}$. Hence the sequent $\boldsymbol{\Gamma}^{\mathbf{s}} \triangleright \boldsymbol{\Delta}^{\mathbf{s}}$ is not $\mathbf{N T R}$-valid, or, in other words, $\Gamma^{\mathrm{s}} \not \nvdash_{\mathrm{NTR}} \boldsymbol{\Delta}^{\mathrm{s}}$.

\section{$3 \quad$ Adding $\rightarrow$ to the object language: the logic NTR $\rightarrow$}

\subsection{Syntactic definition of $\mathrm{NTR}^{\rightarrow}$}

The logic $\mathbf{N} \mathbf{T R} \rightarrow$ will be a set of theorems in the language with propositional letters $p, q, r, s, t, p_{1}, p_{2}, \ldots$ and logical symbols $\rightarrow, \vee$ and $\neg$. The logic is only defined syntactically. We have not yet devised a direct semantics like the one for NTR.

The only symbol we add is $\rightarrow$. We formalize this symbol by means of the most straight forward implication introduction rules, to make it exactly reflect the metatheoretic relevant CL-implication. The other rules are exactly the same as the ones for NTR.

Definition 5. Syntactic consequence. Where $A$ is a formula the only logical 
symbols in which are $\rightarrow, \vee$, and $\neg, \vdash_{\mathbf{N T R}} \rightarrow A$ iff the sequent $\triangleright A$ is derivable by means of the rules and axioms listed below.

The only axiom schema:

$$
A \triangleright A
$$

The only structural rules:

$$
\frac{\boldsymbol{\Gamma}, A, A \triangleright \boldsymbol{\Delta}}{\boldsymbol{\Gamma}, A \triangleright \boldsymbol{\Delta}} \mathrm{LCON}_{\mathbf{\Gamma} \triangleright A, A, \boldsymbol{\Delta}}^{\mathrm{\Gamma CON}}
$$

The rules for $\neg$ :

$$
\frac{\Gamma, A \triangleright \boldsymbol{\Delta}}{\Gamma \triangleright \neg A, \boldsymbol{\Delta}} \mathrm{R} \quad \frac{\boldsymbol{\Gamma} \triangleright A, \boldsymbol{\Delta}}{\boldsymbol{\Delta}, \neg A \triangleright \boldsymbol{\Delta}}\llcorner
$$

The rules for $\vee$ :

$$
\frac{\boldsymbol{\Gamma} \triangleright A, \boldsymbol{\Delta}}{\boldsymbol{\Gamma} \triangleright A \vee B, \boldsymbol{\Delta}} \mathrm{R \vee 1} \quad \frac{\boldsymbol{\Gamma} \triangleright B, \boldsymbol{\Delta}}{\boldsymbol{\Gamma} \triangleright A \vee B, \boldsymbol{\Delta}} \mathrm{R \vee 2} \quad \frac{\boldsymbol{\Gamma}_{\mathbf{1}}, A \triangleright \boldsymbol{\Delta}_{\mathbf{1}} \quad \boldsymbol{\Gamma}_{\mathbf{2}}, B \triangleright \boldsymbol{\Delta}_{2}}{\boldsymbol{\Gamma}_{1}, \boldsymbol{\Gamma}_{2}, A \vee B \triangleright \boldsymbol{\Delta}_{1}, \boldsymbol{\Delta}_{2}} \mathrm{~L} \vee
$$

The rules for $\rightarrow$ :

$$
\frac{\boldsymbol{\Gamma} A \triangleright B, \boldsymbol{\Delta}}{\Gamma \triangleright A \rightarrow B, \boldsymbol{\Delta}} \mathrm{R} \rightarrow \quad \frac{\boldsymbol{\Gamma}_{\mathbf{1}} \triangleright A, \boldsymbol{\Delta}_{\mathbf{1}} \quad \boldsymbol{\Gamma}_{\mathbf{2}}, B \triangleright \boldsymbol{\Delta}_{2}}{\boldsymbol{\Gamma}_{1}, \boldsymbol{\Gamma}_{2}, A \rightarrow B \triangleright \boldsymbol{\Delta}_{1}, \boldsymbol{\Delta}_{2}} \mathrm{~L} \rightarrow
$$

All derived rules for $\mathbf{N T R}$ mentioned in the last section are also derivable in $\mathbf{N T R} \rightarrow$. In the examples we will use them with same names.

We obtain a set of formulas $A$ in the language with logical symbols $\neg$, $\vee$ and $\rightarrow$ such that $\vdash_{\mathbf{N T R}} \rightarrow A$. Those are the theorems that formalize the relevant classical logic implication relation by means of the symbol $\rightarrow$ in the object language.

\subsection{Examples of NTR $\rightarrow$-proofs}

We give a couple of examples of $\mathbf{N T R}{ }^{\rightarrow}$-proof trees.

The first example is the Distributivity (also called Distribution) of conjunction and disjunction. We mention this rule in particular because the incomplete sequent calculus for $\mathbf{R}$ called $\mathbf{L R}$ (see below) is unable to prove this. The fact that we have more permissive rules for $\vee$ or $\wedge$ enables us to derive Distributivity without complications.

Australasian Journal of Logic (16:2) 2019 Article no. 1 


$$
\begin{aligned}
& \frac{q \triangleright q \quad s \triangleright s}{q \vee s \triangleright q, s} \mathrm{~L} \vee \quad \frac{q \triangleright q \quad r \triangleright r}{q \vee r \triangleright q, r} \mathrm{~L} \vee \quad \frac{p \triangleright p \quad s \triangleright s}{p \vee s \triangleright p, s} \mathrm{~L} \vee \quad \frac{p \triangleright p \quad r \triangleright r}{p \vee r \triangleright p, r} \mathrm{~L} \vee
\end{aligned}
$$

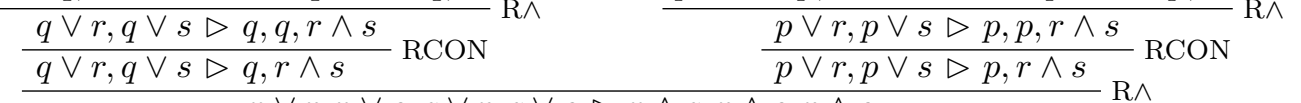

$$
\begin{aligned}
& \begin{array}{c}
\frac{p \vee r, p \vee s, q \vee r, q \vee s \triangleright p \wedge q, r \wedge s, r \wedge s}{p \vee r, p \vee s, q \vee r, q \vee s \triangleright p \wedge q, r \wedge s} \mathrm{RCON} \\
\frac{\mathrm{p} \vee r, p \vee s, q \vee r, q \vee s \triangleright(p \wedge q) \vee(r \wedge s)}{(p \vee r) \wedge(p \vee s) \wedge(q \vee r) \wedge(q \vee s) \triangleright(p \wedge q) \vee(r \wedge s)} \mathrm{R} \wedge \mathrm{m}
\end{array} \\
& \frac{\frac{(p \vee r) \wedge(p \vee s) \wedge(q \vee r) \wedge(q \vee s) \triangleright(p \wedge q) \vee(r \wedge s)}{} \mathrm{L} \wedge \mathrm{m}}{\triangleright((p \vee r) \wedge(p \vee s) \wedge(q \vee r) \wedge(q \vee s)) \rightarrow((p \wedge q) \vee(r \wedge s))} \mathrm{R} \rightarrow
\end{aligned}
$$

The next proof tree is for a relevant implication in which Disjunctive Syllogism is used. The logic $\mathbf{R}$ does not have this as a tautology. But because of the permissive rule $L \vee$ the implication is derivable in $\mathbf{N T R} \rightarrow$.

$$
\begin{aligned}
& \frac{\frac{p \triangleright p \quad q \triangleright q}{p \vee q \triangleright p, q} \mathrm{~L}}{\mathrm{~L} \neg} \\
& \frac{\frac{p \vee q, \neg p \triangleright q}{p \vee q, \neg p \triangleright q \vee s} \mathrm{R} \vee 1}{} \\
& \frac{\frac{p \vee q, \neg p \wedge r \triangleright q \vee s}{\mathrm{~L} \wedge 1}}{p \vee q \triangleright(\neg p \wedge r) \rightarrow(q \vee s))} \mathrm{R} \rightarrow \\
& \frac{p \vee q \triangleright(\neg p \wedge r) \rightarrow(q \vee s))}{\triangleright \rightarrow} \rightarrow
\end{aligned}
$$

Also the next $\mathbf{N T R}{ }^{\rightarrow}$-proof tree is not valid in $\mathbf{R}$. This time the difference lies in the rule $\mathrm{R} \wedge$, which is not valid in $\mathbf{L R}$.

$$
\frac{\frac{p \triangleright p \quad q \triangleright q}{p, q \triangleright p \wedge q} \mathrm{R} \wedge}{\frac{p \triangleright q \rightarrow(p \wedge q)}{\triangleright p \rightarrow(q \rightarrow(p \wedge q))}} \mathrm{R} \rightarrow
$$

Finally we give an example of a situation in which we can derive a classical logical tautology containing material implications $\supset$ (just like $\mathbf{R}$, also $\mathbf{N T R} \rightarrow$ contains all classical tautologies) but we cannot derive the version with relevant instead of material implications. For good reasons: $q$ is not relevant/useful in arguments proving $p$ from $p$.

Australasian Journal of Logic (16:2) 2019 Article no. 1 


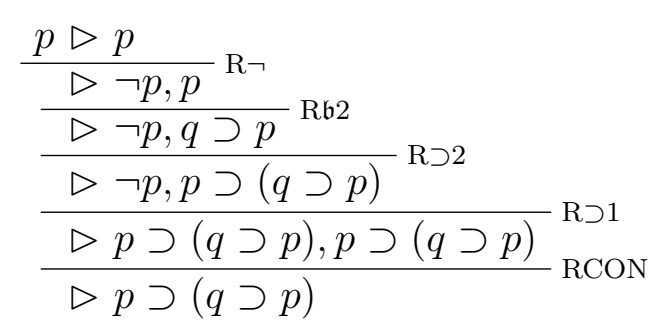

\section{Properties of $\mathrm{NTR}^{\rightarrow}$ : relations with classi- cal and traditional relevance logics}

\subsection{General properties}

We list some easily verifiable properties of $\mathbf{N T R} \rightarrow$.

1. $\vdash_{\mathbf{N T R}} \rightarrow\left(A_{1} \rightarrow\left(A_{2} \rightarrow \ldots\left(A_{n-1} \rightarrow\left(A_{n} \rightarrow B\right) \ldots\right)\right.\right.$ iff $A_{1}, \ldots, A_{n} \vdash_{\mathbf{N T R}}$ $B$. ( $\rightarrow$ captures exactly $\vdash_{\mathbf{N T R}}$ in the $\mathbf{N T R} \rightarrow$ object language).

2. If $\vdash_{\mathbf{C L}} A$ then $\vdash_{\mathbf{N T R}} \rightarrow A$. (all classical tautologies are represented)

3. if $A$ is consistent (i.e. $A \nvdash_{\mathbf{C L}}$ ), $B$ is not tautological (i.e. $\nvdash_{\mathbf{C L}} B$ ) and $A \vdash_{\mathbf{C L}} B$, then $\vdash_{\mathbf{N T R}} \rightarrow A \rightarrow B$. (NTR $\rightarrow$ captures the full consistent and non-tautological part of classical logic).

4. If $\vdash_{\mathbf{N T R}} \rightarrow A \rightarrow B$, then $A \vdash_{\mathbf{F D E}} B$ and $\vdash_{\mathbf{R}} A \rightarrow B$, but $p \wedge(\neg p \vee q) \nvdash_{\mathbf{F D E}}$ $q$ while $\vdash_{\mathbf{N T R}} \rightarrow(p \wedge(\neg p \vee q)) \rightarrow q$, whenever $A$ and $B$ are arrow-free formulas (The implication of $\mathbf{N T R} \rightarrow$ is strictly stronger than the one of $\mathbf{R}$ and $\mathbf{F D E}$, when linking formulas).

5. $\vdash_{\mathbf{N T R}} \rightarrow$ is decidable (in the next Section we show how to reduce $\mathbf{N T R} \rightarrow$ proofs to $\mathbf{L R}$-proofs and the latter is decidable).

6. $A, \neg A \nvdash_{\mathbf{N T R}} B$ and $\nvdash_{\mathbf{N T R}} \rightarrow(A \wedge \neg A) \rightarrow B$ for an arbitrary unrelated $B$. (NTR and $\mathbf{N T R} \rightarrow$ are paraconsistent, but remark that $A, \neg A \vdash_{\mathbf{N T R}}$ ) 


\subsection{The relevance logic $R$}

In this subsection we simply define and describe the well known relevance $\operatorname{logic} \mathbf{R}$ for future reference. TR has as theorems (the formulas $A$ such that $\vdash_{\mathbf{R}} A$ ) the formulas derivable from the following axioms and rules (see [2]). Axioms:

(A1) $\quad A \rightarrow A$

(A2) $\quad(A \rightarrow B) \rightarrow((B \rightarrow C)) \rightarrow(A \rightarrow C))$

(A3) $\quad A \rightarrow((A \rightarrow B) \rightarrow B)$

(A4) $\quad(A \rightarrow(A \rightarrow B)) \rightarrow(A \rightarrow B)$

(A5) $\quad(A \wedge B) \rightarrow A$

$(\mathrm{A} 6) \quad(A \wedge B) \rightarrow B$

(A7) $\quad((A \rightarrow B) \wedge(A \rightarrow C)) \rightarrow(A \rightarrow(B \wedge C))$

(A8) $\quad A \rightarrow(A \vee B)$

(A9) $B \rightarrow(A \vee B)$

$(\mathrm{A} 10) \quad((A \rightarrow C) \wedge(B \rightarrow C)) \rightarrow((A \vee B) \rightarrow C)$

$(\mathrm{A} 11) \quad(A \wedge(B \vee C)) \rightarrow((A \wedge B) \vee C)$

$(\mathrm{A} 12) \quad(A \rightarrow \neg B) \rightarrow(B \rightarrow \neg A)$

(A13) $\neg \neg A \rightarrow A$

Rules:

(R13) from $A$ and $A \rightarrow B$ conclude $B$

(R13) from $A$ and $B$ conclude $A \wedge B$

The logic $\mathbf{R}$ has a well known adequate possible world semantic: the so called Routley-Meyer semantics (cf. [15]). This semantics has a very interesting philosophical interpretation due to Mares (cf. [12]). There is no need to give the definition of the Routley-Meyer models here, nor to explain their philosophical interpretation. For our purposes it suffices that there is such a semantics and a philosophical interpretation. Let $\vDash_{\mathbf{R}} A$ denote that $A$ is valid according to the Routley-Meyer semantics (i.e. $A$ is true in all normal Routley-Meyer worlds).

There is an incomplete sequent calculus for $\mathbf{R}$, the system $\mathbf{L R}$ (short for lattice- $\mathbf{R}$, see [9]). The only reason why it is incomplete is that it does not account for the distributivity of disjunction and conjunction.

Definition 6. Syntactic consequence. Where $A$ is a formula the only logical symbols in which are $\rightarrow, \vee$, and $\neg, \vdash_{\mathbf{L R}} A$ iff the sequent $\triangleright A$ is derivable by means of the rules and axioms listed below.

Australasian Journal of Logic (16:2) 2019 Article no. 1 
The only axiom schema:

$$
A \triangleright A
$$

The only structural rules:

$$
\frac{\boldsymbol{\Gamma}, A, A \triangleright \boldsymbol{\Delta}}{\boldsymbol{\Gamma}, A \triangleright \boldsymbol{\Delta}} \mathrm{LCON} \quad \frac{\boldsymbol{\Gamma} \triangleright A, A, \boldsymbol{\Delta}}{\boldsymbol{\Gamma} \triangleright A, \boldsymbol{\Delta}} \mathrm{RCON}
$$

The rules for $\neg$ :

$$
\left.\left.\frac{\Gamma, A \triangleright \boldsymbol{\Delta}}{\Gamma \triangleright \neg A, \boldsymbol{\Delta}} \mathrm{R}\right\urcorner \quad \frac{\Gamma \triangleright A, \boldsymbol{\Delta}}{\boldsymbol{\Delta}, \neg A \triangleright \boldsymbol{\Delta}} \mathrm{L}\right\urcorner
$$

The rules for $\mathrm{V}$ :

$$
\frac{\boldsymbol{\Gamma} \triangleright A, \boldsymbol{\Delta}}{\Gamma \triangleright A \vee B, \boldsymbol{\Delta}} \mathrm{R \vee 1} \quad \frac{\boldsymbol{\Gamma} \triangleright B, \boldsymbol{\Delta}}{\boldsymbol{\Gamma} \triangleright A \vee B, \boldsymbol{\Delta}} \mathrm{R} \vee 2 \quad \frac{\boldsymbol{\Gamma}, A \triangleright \boldsymbol{\Delta} \quad \boldsymbol{\Gamma}, B \triangleright \boldsymbol{\Delta}}{\boldsymbol{\Gamma}, A \vee B \triangleright \boldsymbol{\Delta}} \mathrm{LV}
$$

The rules for $\rightarrow$ :

$$
\frac{\boldsymbol{\Gamma} A \triangleright B, \boldsymbol{\Delta}}{\boldsymbol{\Gamma} \triangleright A \rightarrow B, \boldsymbol{\Delta}} \mathrm{R} \rightarrow \frac{\boldsymbol{\Gamma}_{\mathbf{1}} \triangleright A, \boldsymbol{\Delta}_{\mathbf{1}} \quad \boldsymbol{\Gamma}_{\mathbf{2}}, B \triangleright \boldsymbol{\Delta}_{2}}{\boldsymbol{\Gamma}_{1}, \boldsymbol{\Gamma}_{2}, A \rightarrow B \triangleright \boldsymbol{\Delta}_{1}, \boldsymbol{\Delta}_{2}} \mathrm{~L} \rightarrow
$$

We have left out the Cut-rule, because there is Cut-elimination in LR.

$\mathbf{R}$ is not decidable, but the $\mathbf{L R}$-fragment is. For a discussion of this property and references to the appropriate literature (by, among others, Saul Kripke and Alasdair Urquhart) see [9, Section 4].

\subsection{Relation between $\mathrm{NTR}^{\rightarrow}$ and $\mathrm{R}$ via a translation}

The $\mathbf{R}$-implication is not rich enough to capture what we call the relevant $\mathbf{C L}$-implication. None of the following forms are valid in $\mathbf{R}$, but they express relevant CL-implications (according to our definition) in the object language.

$$
\begin{aligned}
& \nvdash_{\mathbf{R}}(A \wedge(\neg A \vee B)) \rightarrow B \\
& \nvdash_{\mathbf{R}}(B \vee(A \wedge \neg A)) \rightarrow B \\
& \nvdash_{\mathbf{R}} A \rightarrow(B \rightarrow(A \wedge B)) \\
& \nvdash_{\mathbf{R}} A \rightarrow(A \wedge(B \vee \neg B)) \\
& \nvdash_{\mathbf{R}} A \rightarrow((A \wedge B) \vee \neg B)
\end{aligned}
$$

Let $* \neg A=_{d f} A$ and $* B=_{d f} \neg B$, whenever $B$ is not of the form $\neg A$. The translation $\operatorname{tr}: \mathcal{W} \rightarrow \mathcal{W}$ is recursively defined as follows:

Australasian Journal of Logic (16:2) 2019 Article no. 1 
TR1 $\operatorname{tr}(\sigma)=\sigma$, where $\sigma$ is a letter (atomic formula)

TR2 $\operatorname{tr}(A \vee B)=\operatorname{tr}(A) \vee \operatorname{tr}(B)$

TR3 $\operatorname{tr}(\neg(A \vee B))=\neg(\operatorname{tr}(* A) \rightarrow \neg \operatorname{tr}(* B))$

TR4 $\operatorname{tr}(\neg \neg A)=\operatorname{tr}(A)$

TR5 $\operatorname{tr}(A \rightarrow B)=\neg \operatorname{tr}(* A) \rightarrow \operatorname{tr}(B)$

TR6 $\operatorname{tr}(\neg(A \rightarrow B))=\neg(\operatorname{tr}(A) \rightarrow \neg \operatorname{tr}(* B))$

The idea behind the translation is that the implication and the negation of $\mathbf{R}$ and of $\mathbf{N T R} \rightarrow$ function in exactly the same way. The differences lie in the behaviour of the disjunctions and conjunctions. Disjunctions on the left hand side of $\triangleright$ in $\mathbf{N T R} \rightarrow$ work like intensional disjunctions $\vee^{i}$ in $\mathbf{R}$ $\left(A \vee^{i} B=_{d f} \neg A \rightarrow B\right)$ while disjunctions on the right hand side of $\triangleright$ in $\mathbf{N T R}^{\rightarrow}$ work like extensional disjunctions $\vee^{e}$ in $\mathbf{R}\left(A \vee^{e} B=_{d f} A \vee B\right)$ Conjunctions in antecedents of $\rightarrow$ in NTR work like extensional conjunctions $\wedge^{e}$ in $\mathbf{R}\left(A \wedge^{e} B=_{d f} \neg\left(\neg A \vee^{e} \neg B\right)\right)$ while conjunctions in consequents of $\rightarrow$ work like intensional conjunctions $\wedge^{i}$ in $\mathbf{R}\left(A \wedge^{i} B=_{d f} \neg\left(\neg A \vee^{i} \neg B\right)\right)$.

Theorem 3. Adequacy of the translation. $\vdash_{\mathbf{N T R}} A$ iff $\vDash_{\mathbf{R}} \operatorname{tr}(A)$

Proof. First observe that the fragment of the language of $\mathbf{R}$ without formulas with conjunctions or negations of disjunctions as positive parts suffices to capture all translated formulas. Distributivity of conjunction and disjunction therefore plays no role here. So for this fragment of the language the sequent calculus $\mathbf{L R}$ is complete w.r.t. $\mathbf{R}$. The only difference between the sequent calculus of $\mathbf{N T R} \rightarrow$ and that of $\mathbf{L R}$ is the rule $L \vee$. In NTR this rule is replaced by what would be the derived rule ${ }^{7}$ for left introduction of intensional disjunction in $\mathbf{L R}$ :

$$
\frac{\boldsymbol{\Gamma}_{\mathbf{1}}, A \triangleright \boldsymbol{\Delta}_{\mathbf{1}} \quad \boldsymbol{\Gamma}_{\mathbf{2}}, B \triangleright \boldsymbol{\Delta}_{2}}{\boldsymbol{\Gamma}_{1}, \boldsymbol{\Gamma}_{2}, A \vee^{i} B \triangleright \boldsymbol{\Delta}_{1}, \boldsymbol{\Delta}_{2}}
$$

So, to obtain a correct $\mathbf{L R}$-proof we simply need to use the rule $\mathrm{LV}^{i}$ instead of $\mathrm{LV}$. This is exactly what the translation does: it translates disjunctions into intensional disjunctions in subformulas that end up on the left hand side of $\triangleright$.

$$
{ }^{7} \text { The rule can be derived as follows in LR: } \frac{\left.\frac{\boldsymbol{\Gamma}_{\mathbf{1}}, A \triangleright \boldsymbol{\Delta}_{\mathbf{1}}}{\boldsymbol{\Gamma}_{\mathbf{1}} \triangleright \neg A, \boldsymbol{\Delta}_{\mathbf{1}}} \mathrm{R}\right\urcorner \boldsymbol{\Gamma}_{\mathbf{2}}, B \triangleright \boldsymbol{\Delta}_{2}}{\frac{\boldsymbol{\Gamma}_{1}, \boldsymbol{\Gamma}_{2}, \neg A \rightarrow B \triangleright \boldsymbol{\Delta}_{1}, \boldsymbol{\Delta}_{2}}{\boldsymbol{\Gamma}_{1}, \boldsymbol{\Gamma}_{2}, A \vee^{i} B \triangleright \boldsymbol{\Delta}_{1}, \boldsymbol{\Delta}_{2}} \operatorname{def}^{i}} \mathrm{i} .
$$

Australasian Journal of Logic (16:2) 2019 Article no. 1 
We do not have a reverse translation, i.e. it is principally impossible to translate all $\mathbf{R}$ (non-)tautologies into $\mathbf{N T R}$ (non-)tautologies (NTR is decidable, whereas $\mathbf{R}$ is not), but we do have an equivalence result for part of R's language.

Theorem 4. if $A$ is a formula without negative occurrences of $\vee, \vDash_{\mathbf{N T R}} A$ iff $\vDash_{\mathbf{R}} A$

This can be proven by a straight forward induction on the complexity of formulas. It suffices to observe that one does not need TR4.3 for the fragment without negative disjunctions. One can prove that $\operatorname{tr}(A)=A$ whenever this particular clause is not needed.

Via this translation one can indirectly provide the logic $\mathbf{N T R} \rightarrow$ with a possible world semantics (using the possible world semantics of $\mathbf{R}$ ). Doing this is technically a bit tedious but does not involve any difficulties. It is however still unclear how to interpret this semantics philosophically. We were not able to find a good reason why one would, given this semantics, interpret disjunctions differently depending on the side of the implication on which they occur.

There are however promising outlooks for an exact truthmaker semantics (in the vain of Kit Fine's work, e.g. [10]) of NTR in terms of possible situations instead of possible worlds. It is an exact semantics in Fine's sense: a possible situation only makes a sentence true if the whole situation is relevant for the sentence.

\subsection{Relation with Classical Relevance}

The only difference between the logic $\mathbf{N T R} \rightarrow$ and the logic $\mathbf{R R}$ defined in [22] is that in $\mathbf{N T R} \rightarrow$ we can derive a relevant implication from a material implication, i.e. we have $(\neg A \vee B) \rightarrow(A \rightarrow B)$ as an $\mathbf{N T R} \rightarrow$-theorem. Because, from an external perspective, this may be seen as a fallacious inference, $\mathbf{R R}$ was designed in such a way that such theorems are avoided. This is done by not translating $\mathbf{R} \mathbf{R}$ into $\mathbf{R}$ but into the logic $\mathbf{R} \mathbf{2}$, which is $\mathbf{R}$ but with two non-equivalent relevant $\mathbf{R}$-implications $\mapsto$ and $\rightarrow$, by means of the following translation function:

$$
\begin{aligned}
& \operatorname{tr} R R(\sigma)=\sigma, \text { where } \sigma \text { is a sentential letter, } \\
& \operatorname{tr} R R(A \vee B)=\operatorname{tr} R R(A) \vee \operatorname{tr} R R(B), \\
& \operatorname{tr} R R(A \rightarrow B)=* \operatorname{tr} R R(* A) \mapsto \operatorname{tr} R R(B),
\end{aligned}
$$

Australasian Journal of Logic (16:2) 2019 Article no. 1 


$$
\begin{aligned}
& \operatorname{tr} R R(\neg(A \vee B))=\neg(\operatorname{tr} R R(* A) \rightarrow * \operatorname{tr} R R(* B)), \\
& \operatorname{tr} R R(\neg \neg A)=\operatorname{tr} R R(A), \text { and finally } \\
& \operatorname{tr} R R(\neg(A \rightarrow B))=\neg(\operatorname{tr} R R(A) \mapsto * \operatorname{tr} R R(* B)) .
\end{aligned}
$$

If we now consider that $\rightarrow$ is just the standard relevant implication of $\mathbf{R R}$ (for which we use simply $\rightarrow$ in $\mathbf{N T R} \rightarrow$ ), we can see that this translation function is exactly the same as the function $t r$, as soon as we conflate the two relevant $\mathbf{R}$-implications $\mapsto$ and $\rightarrow$ into the regular $\mathbf{R}$-implication.

The result of this is that $\mathbf{N T R} \rightarrow$ is at least as strong (and actually stronger given that $\vdash_{\mathbf{N T R}}(\neg A \vee B) \rightarrow(A \rightarrow B)$ but not $\vdash_{\mathbf{R R}}(\neg A \vee B) \rightarrow$ $(A \rightarrow B))$ if we compare the logics by letting $\rightarrow$ correspond to $\rightarrow$.

Although the presented logics in the present paper are very related to those defined in [22], this paper's sequent calculus and its general, logicindependent definition of relevance are entirely original.

\section{Conclusion}

In this paper we have first presented and argued for a stipulative definition of what a relevant $\mathbf{L}$-implication is, for a Tarskian consequence relation $\mathbf{L}$. We have explained that the definition is based on being faithful to the logic $\mathbf{L}$, on the formality of relevant implication, and on the idea that premises and conclusions have to be useful in some argument for the implication in order for them to be relevant for the implication.

Then we have developed a sound and complete sequent calculus for relevant $\mathbf{C L}$-implication $\vDash_{\mathbf{N T R}}$. We have provided the required metaproofs and have given some examples of proof trees.

Subsequently, we have added an implication $\rightarrow$ to NTR that reflects exactly the relevant meta-implication $\vDash_{\mathbf{N T R}}$ in the object language. Arguably the resulting logic $\mathbf{N T R} \rightarrow$ is a relevance logic in the traditional sense of the word defining a set of theorems that formalize relevant implication.

Finally, we have listed some properties of the new logic NTR $\rightarrow$. (Among other features) $\mathbf{N} \mathbf{T R} \rightarrow$ turns out to have three useful properties the combination of which seems counterintuitive: (1) It has classical richness in case the antecedent is consistent and the consequent non-tautological; so it does validate i.e. disjunctive syllogism $\left(\vdash_{\mathbf{N T R}} \rightarrow(p \wedge(\neg p \vee q)) \rightarrow q\right)$. (2) It is relevant in a reasonable sense (so it has the variable sharing property, etc.). (3) It is decidable (if one starts from the sequent that should be proven,

Australasian Journal of Logic (16:2) 2019 Article no. 1 
in a finite time one can find all possible proofs by applying the rules in reverse, which results each time in less complex sequents). The combination of these three properties is only possible because the calculus has no cut rule and so the formalized relevant implication is not transitive $\left(\vdash_{\mathbf{N T R}} \rightarrow A \rightarrow B\right.$ and $\vdash_{\mathbf{N T R}} \rightarrow B \rightarrow C$ does not necessarily entail $\left.\vdash_{\mathbf{N T R}} \rightarrow A \rightarrow C\right)$. This may seem suspicious in light of $99 \%$ of the literature on relevant implication, but we have given arguments to the effect that one should not expect relevant implication to be transitive in the first place.

It should be noted that the system $\mathbf{N T R} \rightarrow$ shows some resemblances with Neil Tennant's not fully transitive core logic (cf. for example [18, 19, 20]) and with truth-relevance as proposed by Richard Díaz (cf. [7]). However the presented system is quite different from these accounts. Tennant proposes a relevant consequence relation that is classical in similar situations (consistent premises, conclusion not tautological) as NTR, but does not define a system of relevant implication, a sequent calculus, or a notion of relevance defined by means of notions like non-redundancy and abstraction. Díaz uses techniques similar to the ones presented here to achieve relevance by pruning tableaux trees such that each atom needs to be matched by another one without redundancy (cf. our completeness proof). However he does not develop a sequent calculus and his system is not classical in the sense that, in his approach, each symbol becomes relevant/intensional ( $\vee$ just as much as $\rightarrow$ ), which results in a serious reduction of the classical (arrow-free) tautologies. By contrast, $\mathbf{N T R}^{\rightarrow}$ proves all (arrow-free) classical tautologies.

The logic we have presented here is a first step in a long term philosophical project. The idea behind this project is that notions of relevance occurring in philosophy (the requirement of relevance of explanans for explanandum in theories of explanation, the requirement of relevance of the antecedent for the consequent in standard counterfactuals, relevance in justifications, grounding, imagination, abduction etc.) could be unified by a relevance logic if that logic is sufficiently close to the deductive logic that is preferred in that domain of philosophy for independent reasons (mostly classical, intuitionistic, or paraconsistent logic). To execute this project, future logical work will include the elaboration of a predicative (quantified) version of NTR, proof theories of our notion of relevance for other logics than classical logic and a truthmaker semantics for NTR, NTR $\rightarrow$, and for non-classical versions.

Australasian Journal of Logic (16:2) 2019 Article no. 1 


\section{References}

[1] Wilhelm Ackermann. Begründung einer strengen implikation. Journal of Symbolic Logic, 21:113-128, 1956.

[2] Alan R. Anderson and Nuel D. Belnap. Entailment: The Logic of Relevance and Necessity, volume 1. Princeton University Press, Princeton, 1975 .

[3] Alan Ross Anderson, Nuel D. Belnap, and J. Michael Dunn. Entailment: The Logic of Relevance and Necessity, volume 2. Princeton University Press, Princeton, 1992.

[4] Arnon Avron. Whither relevance logic? Journal of Philosophical Logic, 21(3):243-281, 1992.

[5] John P Burgess. Philosophical logic. Princeton University Press, 2009.

[6] Alonzo Church. The weak positive implication calculus. Journal of Symbolic Logic, 16:238, 1951.

[7] M. Richard Díaz. Topics in the logic of relevance. Philosophia Verlag Gmbh, München, 1981.

[8] J. Michael Dunn. The Algebra of Intensional Logics. PhD thesis, University of Pittsburgh, 1966.

[9] J. Michael Dunn and Greg Restall. Relevance logic. In Dov M. Gabbay, editor, Handbook of Philosophical Logic, volume 6, pages 1-136. Kluwer Academic Publishers, second edition, 2002.

[10] Kit Fine. Truthmaker semantics. In A Companion to the Philosophy of Language, chapter 22, pages 556-577. Wiley-Blackwell, 2017.

[11] Lloyd Humberstone. The Connectives. MIT press, Cambridge, Massachusetts, 2011.

[12] Edwin D Mares. Relevant logic: A philosophical interpretation. Cambridge University Press, 2004.

[13] Robert K. Meyer and Richard Routley. Classical relevant logics II. Studia Logica, 33:183-194, 1973.

Australasian Journal of Logic (16:2) 2019 Article no. 1 
[14] Stephen Read. Relevant logic: a philosophical examination of inference. Basil Blackwell, Oxford, 1988.

[15] Richard Routley and Robert K. Meyer. Semantics of entailment. In Hugues Leblanc, editor, Truth Syntax and Modality, pages 194-243. North Holland, 1973.

[16] Richard Routley, Val Plumwood, Robert K. Meyer, and Ross T. Brady. Relevant Logics and their Rivals. Ridgeview, 1982.

[17] T. J. Smiley. Entailment and deducibility. Proceedings of the Aristotelian Society, 59:233-254, 1958.

[18] Neil Tennant. Perfect validity, entailment and paraconsistency. Studia Logica, 43(1):181-200, 1984.

[19] Neil Tennant. The transmission of truth and the transitivity of deduction. In Dov Gabbay, editor, What is a Logical System?, volume 4 of Studies in Logic and Computation, pages 161-177. Oxford University Press, Oxford, 1994.

[20] Neil Tennant. Relevance in reasoning. In Stewart Shapiro, editor, Handbook of Philosophical Logic and Mathematics, pages 696-726. Oxford University Press, Oxford, 2004.

[21] Alasdair Urquhart. The Semantics of Entailment. PhD thesis, University of Pittsburgh, 1972.

[22] Peter Verdée and Inge De Bal. A new approach to classical relevance. Studia Logica, 103(5):919-954, 2015.

Australasian Journal of Logic (16:2) 2019 Article no. 1 